\title{
HOMOLOGICAL ALGEBRA ON A COMPLETE INTERSECTION, WITH AN APPLICATION TO GROUP REPRESENTATIONS
}

\author{
BY \\ DAVID EISENBUD ${ }^{1}$
}

\begin{abstract}
Let $R$ be a regular local ring, and let $A=R /(x)$, where $x$ is any nonunit of $R$. We prove that every minimal free resolution of a finitely generated $A$-module becomes periodic of period 1 or 2 after at most $\operatorname{dim} A$ steps, and we examine generalizations and extensions of this for complete intersections. Our theorems follow from the properties of certain universally defined endomorphisms of complexes over such rings.
\end{abstract}

Let $A$ be a commutative ring, and let $x \in A$ be a nonzero divisor. How does homological algebra over $A /(x)=B$ differ from that over $A$ ? In this paper we will study a certain natural endomorphism $t$ of complexes of free $A /(x)$-modules which seems to reflect some of the difference. For example, the (homotopic) triviality of $t$ is an obstruction (closely related to the usual one in Ext $_{B}^{2}$ ) to the lifting of a complex of free $B$-modules to a complex of free $A$-modules. More generally, if $x_{1}, \ldots, x_{n}$ is an $A$-sequence, we study $n$ natural endomorphisms $t_{1}, \ldots, t_{n}$ of complexes of free $A /\left(x_{1}, \ldots, x_{n}\right)$-modules, and try to use them to explain the way in which free resolutions over $A /\left(x_{1}, \ldots, x_{n}\right)$ differ from free resolutions over $A$ (the construction and elementary properties of these endomorphisms is given in §1).

In this paper, we will study the case in which $A$ is a regular local ring and $B=A /\left(x_{1}, \ldots, x_{n}\right)$ is not regular. (It would also be very interesting to understand the case in which both $A$ and $A /(x)=B$ were regular-with, say, $A$ of mixed characteristic and $B$ ramified or of characteristic $p$.) In this case, the homological algebra over $A$ is dominated, roughly speaking, by the fact that minimal $A$-free resolutions are finite; we seek to understand the eventual behavior of minimal $B$-free resolutions in terms of the $t_{i}$. For example, if $n=1$, so that $B=A /(x)$, we prove that $t$ is eventually an isomorphism, so that every minimal $B$-free resolution becomes periodic of period 2 after at most $1+\operatorname{dim} B$ steps (§6). We also show that the $B$-modules with periodic resolutions are the maximal Cohen-Macaulay modules without free direct summands. Since the periodic part of a periodic resolution over $A /(x)$ (or more generally, over $A /\left(x_{1}, \ldots, x_{n}\right)$, if $x_{1}, \ldots, x_{n}$ is an $A$-sequence) is easy to describe explicitly ( $\$ 5)$, this yields information on maximal CohenMacaulay modules.

Received by the editors February 24, 1978.

AMS (MOS) subject classifications (1970). Primary 13D99, 13H10, 13C05; Secondary $18 \mathrm{H} 20$.

${ }^{1}$ The author is grateful to the Sloan Foundation and the NSF for their support, and to Harvard University and the IHES for their hospitality during the preparation of this work. 
We are further able to apply this theory, in $\$ 9$, to deduce some properties of modular representations of finite groups. (Although $\$ 9$ is last, only the ideas of 1.7 , 3.1, and 5.2 are used there.)

Our results on periodic resolutions, and the case $n=1$, are contained in $\$ \S 5$ and 6. Because they are more elementary and complete than the rest of this paper, the reader may wish to look at these sections first.

For $n>1$ the situation is more complicated. To indicate the nature of our results, we will discuss the contents of the paper by sections. We then mention some of the open problems.

$\S 1$ is devoted to the construction and properties of the $t_{i}$. They are endomorphisms of complexes, of degree -2 . They are defined in terms of certain choices, but are unique and natural up to homotopy, and behave well under change of rings. $\$ 2$ contains an example which is of theoretical importance to us. Here we see that, on the minimal resolution $F$ of the residue class field of $B$, the $t_{i}$ commute, so that $F$ is a $B\left[t_{1}, \ldots, t_{n}\right]$-module. In fact it is a sort of "relatively artinian" module-for instance, if $B$ is artinian, then $\mathbf{F}$ is an artinian $B\left[t_{1}, \ldots, t_{n}\right]$-module. This fact, which should be taken as measuring the nontriviality of the $t_{i}$, is in some sense typical of what we find for minimal resolutions in general; in the next section we use it to show that if the residue class field of $B$ is infinite, then for any minimal $B$-free resolution

$$
\mathbf{F}: \ldots \rightarrow F_{1} \rightarrow F_{0}
$$

there is a linear combination $t$ of the $t_{i}$ which is eventually an epimorphism-that is, $t: F_{i+2} \rightarrow F_{i}$ is an epimorphism for large $i$. This is the result we will use for the analysis of minimal $B$-free resolutions.

One immediate consequence of this result, explored in $\$ 4$, is that if the ranks of the free modules $F_{i}$ are bounded, then in fact $\mathbf{F}$ becomes periodic, of period 2, after finitely many steps; the map $t$ is in this case the isomorphism which gives the periodicity.

What can be said about periodic resolutions of period 2? All the examples of which we are aware (over local rings), and, in particular, all examples over a ring like $B$ (a complete intersection) are constructed in a very simple way. This construction and some of its properties are explored in $\$ 5$. We also prove, there, that in any resolution of period 2, all the ranks of the free modules in the resolution are equal.

$\$ 6$ is devoted to the case $n=1$, where $B=A /(x)$. In this situation every resolution becomes periodic, and periodic resolutions correspond to maximal Cohen-Macaulay $B$-modules. By virtue of the theory developed in $\$ 5$, both these things correspond to factorizations, in a matrix ring over $A$, of $x$ times the identity matrix.

In $\$ 7$ we return to the general case $n>1$; we show how to construct a $B$-free resolution of a $B$-module $M$ from an $A$-free resolution (even without the hypothesis that $A$ is regular). 
In general, the $B$-free resolution obtained by the method of $\$ 7$ is not minimal. However, using the "eventually epimorphic" map $t$ of $\$ 3$, we show in $\$ 8$ that some truncation of any minimal $B$-free resolution is obtained, by the process of $\S 7$, from a resolution over a ring of the form $B_{1}=A /\left(x_{2}, \ldots, x_{n}\right)$; that is, a ring which is in some sense closer to being regular. Thus the maps $t_{i}$ lead to a fairly good "relative-codimension 1" structure theory for minimal $B$-free resolutions.

A number of interesting problems are raised by the foregoing ideas. One is: Why period 2? There are plenty of periodic resolutions with large periods over noncommutative rings. (For instance, if $G$ is a group with Sylow $p$-subgroup $S$, where $p$ is an odd prime, and if $Z /(p)$ is the ring of integers modulo $p$, then the $Z /(p)[G]$ free resolution of $Z /(p)$ (as a trivial module) is periodic if and only if $S$ is cyclic, in which case its period is $2 \mid$ Normalizer $_{G} S|/|$ Centralizer $_{G} S \mid$. Note that the difference of this number from 2 is a sort of measure of the noncommutativity of $Z / p[G]$.) But we know of no similar examples for commutative rings. In fact, we do not even know any bounded free resolution which does not become periodic of period 2.

Conjecture. Let $B$ be a local commutative ring. If $\mathbf{F}: \ldots \rightarrow F_{1} \rightarrow F_{0}$ is a minimal $B$-free resolution such that the ranks of the free modules $F_{i}$ are bounded, then $F$ is eventually periodic of period 2 .

Let us return to the case of a ring $B=A /\left(x_{1}, \ldots, x_{n}\right)$, where $A$ is regular local, and $x_{1}, \ldots, x_{n}$ is an $A$-sequence. We define a standard $B$-free resolution to be one which is constructed, by the method of $\$ 7$, from an $A$-free resolution. For standard resolutions there is a natural choice of the maps $t_{i}$, and these standard $t_{i}$ commute with one another.

Conjecture. The minimal free resolution of any $B$-module is a subcomplex of a standard resolution, in such a way that the maps $t_{i}$ may be chosen to be induced by the standard $t_{i}$. In particular, the maps $t_{i}$ may be chosen to commute.

In the spirit of this paper, it would be interesting to prove this conjecture just for some truncation of any minimal free resolution.

Since the $B$-duals of standard resolutions are free $B\left[t_{1}, \ldots, t_{n}\right]$-modules, one consequence of this conjecture, even in the weakened form above, would be the "finite determination" of any minimal $B$-free resolution.

I am glad to acknowledge the great debt that this paper owes to the important work [G2] of Gulliksen. Suppose $A$ is a local ring, $x_{1}, \ldots, x_{n}$ is an $A$-sequence, and $B=A /\left(x_{1}, \ldots, x_{n}\right)$. Gulliksen defines, for example, a $B\left[t_{1}, \ldots, t_{n}\right]$-module structure on $\operatorname{Tor}^{B}(M, N)$ for any $B$-modules $M$ and $N$, and proves that if $\operatorname{Tor}^{A}(M, N)$ is artinian over $A$, then $\operatorname{Tor}^{B}(M, N)$ is artinian over $B\left[t_{1}, \ldots, t_{n}\right]$. Now consider a $B$-free resolution $F$ of $M$; the endomorphisms $t_{i}$ of $F$ that we define are unique and commutative up to homotopy, so they too define a $B\left[t_{1}, \ldots, t_{n}\right]$-module structure on $\operatorname{Tor}^{A}(M, N)$. These structures are the same [M]. Given this fact, our Lemma 3.2 becomes a special case of Gulliksen's result (the general case could be deduced in much the same way). Since our construction of the $t_{i}$ is somewhat more straightforward than Gulliksen's, our methods offer a simplified approach to Gulliksen's work. 
It should also be noted that some part of our results on hypersurfaces were proved first, in a slightly different form, by Shamash in [Sh], who relied, in turn, partly on results of Nagata [Nag].

I am grateful to $M$. Hochster for a simplified proof of Theorem 6.1. I also profited from discussions with the members of the "ad hoc seminar on periodic phenomena"-Jon Alperin, Harvey Margolis, and Mark Ramras-and with Graham Evans.

0. Some preliminaries. Here we collect various definitions, and one general lemma, that we will use repeatedly.

In this paper, all rings are supposed to be commutative and noetherian, and modules (except graded ones) are finitely generated.

Let $A$ be a ring. The sequence of maps of free $A$-modules

$$
\mathrm{F}: \ldots \stackrel{\partial}{\rightarrow} F_{2} \stackrel{\partial}{\rightarrow} F_{1} \stackrel{\partial}{\rightarrow} F_{0}
$$

is said to be a complex if $\partial^{2}=0$. If $A$ is local with maximal ideal $M$, then $F$ is minimal if $A / M \otimes \partial=0$. A complex $\mathrm{F}$ is a resolution if it is exact except (possibly) at the right-hand end-in this case at $F_{0}$. Any resolution over a local ring is the direct sum of a minimal resolution and various trivial resolutions of the form

$$
\ldots \rightarrow 0 \rightarrow F \stackrel{1}{\rightarrow} F \rightarrow 0 \rightarrow \ldots
$$

(see for example [S, Appendix I to Chapter IV]).

If $\mathbf{F}$ is as above, we write $\mathbf{F}^{(d)}$ for the truncation

$$
F^{(d)}: \ldots \rightarrow F_{d+1} \rightarrow F_{d}
$$

of $\mathbf{F}$ at the $d$ th step.

If $M$ is an $A$-module, then a sequence of elements $x_{1}, \ldots, x_{n} \in A$ is an $M$-sequence if

(a) $x_{i+1}$ is a nonzero divisor on $M /\left(x_{1}, \ldots, x_{i}\right) M$,

(b) $\left(x_{1}, \ldots, x_{n}\right) M \neq M$.

(If $M$ is finitely generated, as we have supposed, and if $A$ is local, then condition (b) only serves to exclude the possibilities $M=0$ and $\left(x_{1}, \ldots, x_{n}\right)=A$.)

LEMMA 0.1. Let $B$ be a local ring, and suppose that

$$
\mathrm{F}: \ldots \rightarrow F_{d+1} \stackrel{\varphi_{d+1}}{\rightarrow} F_{d} \stackrel{\varphi_{d}}{\rightarrow} F_{d-1} \rightarrow \cdots \stackrel{\varphi_{1}}{\rightarrow} F_{0}
$$

is a $B$-free resolution. Then

(i) If $a_{1}, \ldots, a_{d}$ is a $B$-sequence, then $a_{1}, \ldots, a_{d}$ is a regular sequence on image $\varphi_{k}$ for $k \geqslant d$.

(ii) If $\mathbf{F}$ is minimal, then image $\varphi_{k}$ has no free summands for $k>1+\operatorname{depth} B$.

Remark. At least part (i) is well known. Part (ii) is a strengthening of the statement that a $B$-module of finite projective dimension has projective dimension $\leqslant d$.

Proof of Lemma 0.1. Set $B_{m}=B /\left(a_{1}, \ldots, a_{m}\right)$.

To prove part (i), let $k \geqslant d$; by induction we may suppose that $a_{1}, \ldots, a_{d-1}$ is a regular sequence on image $\varphi_{k}$, and it suffices to prove that $a_{d}$ is a nonzero divisor 
on $B_{d-1} \otimes$ image $\varphi_{k}$. However, projective dimension $B_{B} B_{d-1}=d-1$, so

$$
\operatorname{Tor}_{d}\left(B_{d-1}, \operatorname{coker} \varphi_{1}\right)=0 \text {, }
$$

and thus the sequence $0 \rightarrow B_{d-1} \otimes$ image $\varphi_{k} \rightarrow B_{d-1} \otimes F_{k-1}$ is exact; since $a_{d}$ is a nonzero divisor on $B_{d-1}$, this concludes the proof.

For part (ii), suppose that $\Re$ is the maximal ideal of $B$ and that $a_{1}, \ldots, a_{d}$ is a maximal $B$-sequence, so $d=\operatorname{depth} B$.

For $k \geqslant d+1$ we have as before an exact sequence $0 \rightarrow B_{d} \otimes$ image $\varphi_{k} \rightarrow B_{d} \otimes$ $F_{k-1}$. By the minimality of $\mathbf{F}, B_{d} \otimes$ image $\varphi_{k}$ is actually contained in $\Re\left(B_{d} \otimes F_{k-1}\right)$; since $B_{d}$ has depth $0, \mathfrak{R}\left(B_{d} \otimes F_{k-1}\right)$ is annihilated by some nonzero element of $B_{d}$. However, if im $\varphi_{k}$ had a free summand, then $B_{d} \otimes$ im $\varphi_{k}$ would contain a copy of $B_{d}$, which would be a contradiction.

Finally, we will say that a local ring $B$ is a complete intersection (of codimension $<n)$ if it can be written in the form $B=A /\left(x_{1}, \ldots, x_{n}\right)$, where $A$ is regular and $x_{1}, \ldots, x_{n}$ form an $A$-sequence. If $n=1, B$ is said to be a hypersurface. If $B$ is a complete intersection, then it is Gorenstein [B]; in particular, if $\operatorname{dim} B=0$, then $B$ is self-injective.

1. The construction and its naturality. In this section we will construct the endomorphisms of free complexes over a complete intersection which are the fundamental objects of study in this paper.

Let $A$ be a commutative ring, and let $I=\left(x_{1}, \ldots, x_{n}\right)$ be an ideal of $A$. Set $B=A / I$, and suppose that $I / I^{2}$ is a free $B$-module on the images of $x_{1}, \ldots, x_{n}$. (This condition will, for example, be satisfied if $x_{1}, \ldots, x_{n}$ form an $A$-sequence; this is the case of primary interest. In fact if the projective dimension of $I$, as an $A$-module, is finite, then $I / I^{2}$ is free over $B$ if and only if $I$ is generated by an $A$-sequence [L-V].)

Let $\mathrm{F}: \ldots F_{i+2} \stackrel{\partial}{\rightarrow} F_{i+1} \stackrel{\partial}{\rightarrow} F_{i} \rightarrow \ldots$ be a complex of free $B$-modules.

(a) Choose a sequence of free $A$-modules $\tilde{F}_{i}$ and maps $\tilde{\partial}$ between them:

$$
\tilde{\mathbf{F}}: \ldots \tilde{F}_{i+2} \stackrel{\tilde{\partial}}{\rightarrow} \tilde{F}_{i+1} \stackrel{\tilde{\partial}}{\rightarrow} \tilde{F}_{i} \rightarrow \ldots
$$

so that $\mathbf{F}=B \otimes \tilde{\mathbf{F}}$. (To do this, one can think of the maps $\partial$ as being given by matrices over $B$, and define the maps $\tilde{\partial}$ by lifting these matrices to $A$. Of course, in general, $\tilde{\partial}^{2} \neq 0$.)

(b) Since $\tilde{\partial}^{2} \equiv 0$ modulo $\left(x_{1}, \ldots, x_{n}\right)$, we may write $\tilde{\partial}^{2}=\sum x_{j} \tilde{t}_{j}$ where $\tilde{t}_{j}$ : $\tilde{F}_{i} \rightarrow \tilde{F}_{i-2}($ for every $i)$. When necessary, we will write $\tilde{t}_{j}=\tilde{t}_{j}\left(A,\left\{x_{i}\right\}, \tilde{F}\right)$.

Define, for $j=1, \ldots, n$, the map $t_{j}=t_{j}\left(A,\left\{x_{i}\right\}, \mathbf{F}\right): \mathbf{F} \rightarrow \mathbf{F}$ by $t_{j}=B \otimes \tilde{t}_{j}$.

When there is no danger of confusion, we will write $t_{j}(\mathbf{F})$ in place of $t_{j}\left(A,\left\{x_{i}\right\}, F\right)$. We will preserve the above hypothesis and notation throughout this section.

Note. (1) One should perhaps write $t_{j}=t_{j}\left(A,\left\{x_{i}\right\}, \tilde{\mathbf{F}}, \tilde{\partial}\right)$, since $t_{j}$ depends not only on $\mathbf{F}$ but also on the liftings $\tilde{F}_{i}$, $\tilde{\partial}$ chosen. We have chosen to write $\left.t_{j}\left(A,\left\{x_{i}\right\}, F\right\}\right)$, because $t_{j}$ is in fact determined up to homotopy by these data (Corollary 1.4). 
EXAmple. The following is very typical of the case $n=1$; see $\$ \S 2$ and 7 for examples of what happens when $n$ is larger.

Let $A=Z$, the ring of integers, and let $x=x_{1}=8$, so that $B=Z /(8)$. Let $F$ be the minimal free $B$-resolution of the $B$-module $Z /(4)$ :

$$
\text { F: ... } \stackrel{2}{\rightarrow} Z / 8 \stackrel{4}{\rightarrow} Z / 8 \stackrel{2}{\rightarrow} Z / 8 \stackrel{4}{\rightarrow} Z / 8 .
$$

For $\tilde{\mathbf{F}}$, we may choose

$$
\tilde{\mathbf{F}}: \ldots \stackrel{2}{\rightarrow} Z \stackrel{4}{\rightarrow} Z \stackrel{2}{\rightarrow} Z \stackrel{4}{\rightarrow} Z .
$$

Then clearly $\tilde{\partial}^{2}=8=x \cdot 1$, so $\tilde{t}=1$, and

$$
t=t_{1}(Z, 8, F)=1: Z / 8=F_{i+2} \rightarrow F_{i}=Z / 8 \text {. }
$$

Thus the evident periodicity of $F$ is given by $t$.

Proposition 1.1. The map $t_{j}$ is a homomorphism of complexes of degree -2 ; that is $t_{j} \partial=\partial t_{j}$

Proof. We have

$$
\sum_{j} x_{j}\left(\tilde{t_{j}} \tilde{\partial}\right)=\left(\sum_{j} x_{j} \tilde{t}_{j}\right) \tilde{\partial}=\tilde{\partial}^{3}=\tilde{\partial}\left(\sum_{j} x_{j} \tilde{t}_{j}\right)=\sum_{j} x_{j}\left(\tilde{\partial} \tilde{t}_{j}\right) .
$$

Since the elements $x_{j}$ form a free $B$-basis of $I / I^{2}$ we obtain $\tilde{t}_{j} \tilde{\partial} \equiv \tilde{\partial} \tilde{t}_{j}$ modulo $I$, or $t_{j} \partial=\partial t_{j}$.

Proposition 1.2. The map $t_{j}$ is independent of the choice made in (b).

Proof. If, for $j=1, \ldots, n, \tilde{t_{j}^{\prime}}$ is another choice, then $\Sigma_{j} x_{j} \tilde{t}_{j}=\tilde{\partial}^{2}=\Sigma_{j} x_{j} \tilde{t}_{j}^{\prime}$. Thus $\Sigma_{j} x_{j}\left(\tilde{t}_{j}-\tilde{t}_{j}^{\prime}\right)=0$. As in Proposition 1.1 this implies

$$
\tilde{t}_{j}-\tilde{t_{j}^{\prime}} \equiv 0 \text { modulo } I, \quad t_{j}=B \otimes \tilde{t}_{j}=B \otimes \tilde{t}_{j}^{\prime} .
$$

Proposition 1.3 (Naturality). Let

$$
\text { G: } \ldots G_{i+2} \stackrel{\partial^{\prime}}{\rightarrow} G_{i+1} \stackrel{\partial^{\prime}}{\rightarrow} G_{i} \rightarrow \ldots
$$

be another complex of free $B$-modules, and set $s_{j}=t_{j}\left(A,\left\{x_{i}\right\}, \mathbf{G}\right)$. If $f: \mathbf{F} \rightarrow \mathbf{G}$ is a homomorphism of complexes, then for each $j=1, \ldots, n, f t_{j}$ is homotopic to $s_{j} f$.

Proof. Suppose $f$ is of degree $k$, so that $f$ is given by maps $f_{i}: F_{i} \rightarrow G_{i+k}$, for each $i$. Let $(\tilde{\mathbf{F}}, \tilde{\partial})$ and $\left(\tilde{\mathbf{G}}, \tilde{\partial}^{\prime}\right)$ be the sequences of maps of free $A$-modules used in constructing $t_{j}$ and $s_{j}$, and let $\tilde{s}_{j}=\tilde{t}_{j}\left(A,\left\{x_{i}\right\}\right.$, G). Since $f \partial=\partial^{\prime} f$, we may choose maps $\tilde{h}_{j}: \tilde{F}_{i} \rightarrow \tilde{G}_{i+k-1}$, such that $\tilde{f} \tilde{\partial}-\tilde{\partial}^{\prime} \tilde{f}=\sum x_{j} h_{j}$. We have

$$
\begin{aligned}
& \sum x_{j}\left(\tilde{f t_{j}}\right)=\tilde{f} \tilde{\partial}^{2}=\tilde{\partial}^{\prime} \tilde{f} \tilde{\partial}+\sum x_{j} h_{j} \tilde{\partial} \\
& =\tilde{\partial}^{\prime 2} \tilde{f}+\sum x_{j} \tilde{\partial}^{\prime} \tilde{h_{j}}+\sum x_{j} \tilde{h}_{j} \tilde{\partial} \\
& =\sum_{j} x_{j} \tilde{s_{j}} \tilde{f}+\sum_{j} x_{j} \tilde{\partial}^{\prime} \tilde{h}_{j}+\sum_{j} x_{j} \tilde{h_{j}} \tilde{\partial}
\end{aligned}
$$

or,

$$
\sum_{j} x_{j}\left(\tilde{f t_{j}}-\tilde{s_{j}} \tilde{f}\right)=\sum x_{j}\left(\tilde{\partial} \tilde{h}_{j}+\tilde{h_{j}} \tilde{\partial}\right)
$$


If we write $h_{j}=B \otimes \tilde{h}_{j}$, then by the same reasoning as in Proposition 1.1,

$$
f t_{j}-s_{j} f=\partial^{\prime} h_{j}+h_{j} \partial \text {, }
$$

as required.

COROllary 1.4. The maps $t_{j}: \mathbf{F} \rightarrow \mathbf{F}$ are determined uniquely up to homotopy by the data $\left(A,\left\{x_{i}\right\}, F\right)$. In particular, if $M$ is any $B$-module, they induce well-defined maps

$t_{j}: H_{i+2}(\mathrm{~F} \otimes M) \rightarrow H_{i}(\mathrm{~F} \otimes M)$ and $t_{j}: H^{i}(\operatorname{Hom}(\mathrm{F}, M)) \rightarrow H^{i+2}(\operatorname{Hom}(\mathrm{F}, M))$ on the homology and cohomology of $\mathbf{F}$ with coefficients in $M$.

Proof. Apply Proposition 1.3 to the identity map 1: F $\rightarrow$ F.

Corollary 1.5. The maps $t_{j}(j=1, \ldots, n)$ commute up to homotopy.

Proof. Apply Proposition 1.3 to the map $t_{k}: \mathbf{F} \rightarrow \mathbf{F}$.

Problem. Can the maps $t_{j}$ be chosen to commute?

Proposition 1.6. Let $M$ and $N$ be finitely generated $B$-modules, and let $\mathbf{F} \rightarrow M$ and $\mathbf{G} \rightarrow N$ be $B$-free resolutions. Then $t_{j}(\mathbf{F})$ and $t_{j}(\mathbf{G})$ induce the same map on

$$
H_{*}(\mathrm{~F} \otimes N)=\operatorname{Tor}_{*}^{B}(M, N)=H_{*}(M \otimes \mathrm{G}) \text {. }
$$

Thus $\operatorname{Tor}_{*}^{B}(M, N)$ is unambiguously a graded module over the ring $B\left[t_{1}, \ldots, t_{n}\right]$ (with degree $t_{i}=-2$ ).

Remark. By Proposition 1.3, $\operatorname{Tor}_{*}^{B}(M, N)$ is functorial (as a $B\left[t_{1}, \ldots, t_{n}\right]$-module) in $M$ and $N$.

Proof. The isomorphism $H_{*}(\mathrm{~F} \otimes N)=H_{*}(M \otimes G)$ is the composition of isomorphisms on homology induced by the projections of complexes

$$
\mathbf{F} \otimes N \stackrel{\pi_{1}}{\leftarrow} \mathbf{F} \otimes \mathbf{G} \stackrel{\pi_{2}}{\rightarrow} M \otimes \mathbf{G} .
$$

We will prove the proposition by constructing $t_{j}(\mathbf{F} \otimes \mathbf{G})$ in such a way that $\pi_{1} t_{j}(\mathbf{F} \otimes G)=\left(t_{j}(\mathbf{F}) \otimes N\right) \pi_{1}$ and $\pi_{2} t_{j}(\mathbf{F} \otimes \mathbf{G})=\left(M \otimes t_{j}(\mathbf{G})\right) \pi_{2}$.

If $(\tilde{\mathbf{F}}, \tilde{\partial})$ and $(\tilde{\mathbf{G}}, \tilde{\partial})$ are the sequences of maps of free $A$-modules used in the construction of $t_{j}(\mathbf{F})$ and $t_{j}(\mathbf{G})$, then $(\tilde{\mathbf{F}} \otimes \tilde{\mathbf{G}}, \tilde{\partial} \otimes 1+1 \otimes \tilde{\partial})$ is a lifting of $\mathbf{F} \otimes \mathbf{G}$. Note that there is a sign convention for tensor products of graded modules: if $f \in \tilde{F}_{i}$ and $g \in \tilde{\mathbf{G}}$, then $1 \otimes \tilde{\partial}(f \otimes g)=(-1)^{i} f \otimes \tilde{\partial} g$. Because of this sign convention, $1 \otimes \tilde{\partial}$ and $\tilde{\partial} \otimes 1$ anticommute. Thus

$$
(\tilde{\partial} \otimes 1+1 \otimes \tilde{\partial})^{2}=\tilde{\partial}^{2} \otimes 1+1 \otimes \tilde{\partial}^{2}=\sum_{j} x_{j}\left(\tilde{t_{j}}(\tilde{\mathbf{F}}) \otimes 1+1 \otimes \tilde{t}_{j}(\mathbf{G})\right) .
$$

Consequently, we may take $t_{j}(\mathbf{F} \otimes G)=t_{j}(\mathbf{F}) \otimes 1+1 \otimes t_{j}(\mathbf{G})$. This choice has the desired property.

Proposition 1.7 (ChANGe OF RINGS). Let $\alpha: A \rightarrow A^{\prime}$ be a ring homomorphism, and let $x_{1}^{\prime}, \ldots, x_{m}^{\prime}=I^{\prime}$ be an ideal of $A^{\prime}$. Set $B^{\prime}=A^{\prime} / I^{\prime}$, and suppose that $I^{\prime} / I^{\prime 2}$ is $B^{\prime}$-free on the images of $x_{1}^{\prime}, \ldots, x_{n}^{\prime}$. 
Suppose $\alpha(I) \subset I^{\prime}$, with

$$
\alpha\left(x_{i}\right)=\sum_{j=1}^{m} c_{i j} x_{j}^{\prime} \quad \text { for some elements } c_{i j} \in A^{\prime} .
$$

Then $t_{j}\left(A^{\prime},\left\{x_{i}^{\prime}\right\}, B^{\prime} \otimes \mathbf{F}\right)$ is homotopic to $\sum_{i=1}^{n} c_{i j}\left(B^{\prime} \otimes t_{i}\left(A,\left\{x_{i}\right\}, \mathbf{F}\right)\right)$.

Proof. Write $t_{j}=t_{j}\left(A,\left\{x_{i}\right\}, \mathbf{F}\right)$. In view of Corollary 1.4, it is enough to show that with suitable choices in the construction of $t_{j}^{\prime}=t_{j}\left(A^{\prime},\left\{x_{i}^{\prime}\right\}, B^{\prime} \otimes \mathrm{F}\right)$, we will have $t_{j}^{\prime}=\sum_{i=1}^{n} c_{i j}\left(B^{\prime} \otimes t_{i}\right)$. Let $(\tilde{\mathbf{F}}, \tilde{\partial})$ be the sequence of maps of free $A$-modules used in the construction of $t_{j}$, with $\tilde{\partial}^{2}=\sum x_{i} \tilde{t}_{i}$. Take

$$
\left(B^{\prime} \otimes \mathbf{F}\right)^{\sim}=A^{\prime} \otimes \tilde{\mathbf{F}}, \quad\left(B^{\prime} \otimes \partial\right)^{\sim}=A^{\prime} \otimes \tilde{\partial} .
$$

Defining $\tilde{t}_{j}^{\prime}=\sum_{i=1}^{n} c_{i j}\left(A^{\prime} \otimes \tilde{t_{i}}\right)$, we have

$$
\sum_{j} x_{j}^{\prime} \tilde{t}_{j}^{\prime}=\sum_{j} \sum_{i} c_{i j} x_{j}^{\prime}\left(A^{\prime} \otimes \tilde{t}_{i}\right)=\sum_{i} \alpha\left(x_{i}\right)\left(A^{\prime} \otimes \tilde{t}_{i}\right)=A^{\prime} \otimes \tilde{\partial}^{2},
$$

so we may take $t_{j}^{\prime}=t_{j}\left(A^{\prime},\left\{x_{j}^{\prime}\right\}, B^{\prime} \otimes \mathbf{F}\right)=B^{\prime} \otimes \tilde{t}_{j}^{\prime}=B^{\prime} \otimes t_{j}$, as claimed.

The following remarks are due to $\mathrm{V}$. Mehta $[\mathbf{M}]$ :

(1) Consider the case in which $F$ is the free resolution of a $B$-module $M$. The maps $t_{j}$ induce (uniquely defined) natural transformations $t_{j *}: \operatorname{Tor}_{i+2}(M,-) \rightarrow$ $\operatorname{Tor}_{i}(M,-)$ for each $i$. Any such natural transformation comes from an element $\xi_{j} \in \operatorname{Ext}^{2}(M, M)$. These "natural" elements of $\operatorname{Ext}^{2}(M, M)$ are the obstructions to lifting $M$ modulo $\left(x_{1}, \ldots, x_{n}\right)^{2}$ studied by Nastold [Nas], Grothendieck, and others.

(2) The natural transformations $t_{j^{*}}: \operatorname{Tor}_{i+2}(M,-) \rightarrow \operatorname{Tor}_{i}(M,-)$ in (1) coincide, in the case where $x_{1}, \ldots, x_{n}$ are an $A$-sequence, with the ones defined by Gulliksen in [G2].

(3) The results of this section may be summarized by saying that if $I / I^{2}$ is $B=A / I$-free, then the symmetric algebra $\operatorname{Sym}_{B}\left(\operatorname{Hom}_{B}\left(I / I^{2}, B\right)\right)$ acts, functorially in the pair $(A, I)$, on the category of complexes of free $B$-modules modulo homotopy. Mehta has shown that a similar result holds for arbitrary ideals $I$.

2. Example. The resolution of the residue class field. Let $\boldsymbol{A}$ be a regular local ring with maximal ideal $\Re$ and residue class field $K=A / \mathfrak{N}$. Let $x_{1}, \ldots, x_{n} \in \mathfrak{N}^{2}$ be an $A$-sequence, and set $B=A /\left(x_{1}, \ldots, x_{n}\right)$. We are going to compute the minimal $B$-free resolution $F$ of $K$ (following [Tate]) and the maps $t_{i}\left(A,\left\{x_{i}\right\}, F\right)$ of F. In particular, we will see that each $t_{i}$ is an epimorphism. In $\$ 7$, we will generalize the construction to obtain a (nonminimal) $B$-free resolution of any $B$-module, starting from an $A$-free resolution of that module (Theorem 7.2). Also, given any minimal $B$-free resolution, we will show that after a sufficiently general choice of generators $x_{i}$ for the ideal $\left(x_{1}, \ldots, x_{n}\right), t_{1}$ is an epimorphism on some truncation of the resolution (Theorem 3.1).

We begin by describing F; see [G-L] for further details.

Let $y_{1}, \ldots, y_{m}$ be a minimal set of generators for the maximal ideal $\mathfrak{N}$ of $A$. The minimal $A$-free resolution of $K$ is the Koszul complex $\mathbf{K}\left(y_{1}, \ldots, y_{m}\right)$. This complex has the structure of a differential graded algebra, the underlying algebra 
being the exterior algebra $\Lambda A^{m}$, with differential $\partial$, say.

Because $x_{1}, \ldots, x_{n} \in \Re^{2}$, there exist elements $x_{i}^{\prime} \in \mathbf{K}_{1}$ such that $\partial x_{i}^{\prime}=x_{i}$. We will write $\sigma_{i}$ for the map $\mathbf{K}\left(y_{1}, \ldots, y_{m}\right) \rightarrow \mathbf{K}\left(y_{1}, \ldots, y_{m}\right)$ of graded modules given by $\sigma_{i}(e)=e \wedge x_{i}$. It is easy to check that $\sigma_{i} \partial+\partial \sigma_{i}=x_{i} \cdot 1$, so that $\sigma_{i}$ is a homotopy for multiplication by $x_{i}$. Since $\sigma_{i}$ is defined from the exterior product, we have $\sigma_{i} \sigma_{j}=-\sigma_{j} \sigma_{i}, \sigma_{i}^{2}=0$ for all $i, j$.

To construct the minimal $B$-free resolution of $K$, we make use of the divided power algebra $D\left(A^{n}\right)$. As a graded module, $D\left(A^{n}\right)$ is defined as the dual of the symmetric algebra:

$$
D\left(A^{n}\right)=\operatorname{Hom}_{\mathrm{gr} A \text {-modules }}\left(A\left[t_{1}, \ldots, t_{n}\right], A\right) .
$$

(The algebra structure of $D\left(A^{n}\right)$ will not concern us.)

For our purposes, we want to grade $D\left(A^{n}\right)$ in such a way that it is nonzero only in even degrees, so we set degree $t_{i}=2$ for all $i . D\left(A^{n}\right)$ is, from its definition, clearly a module over $A\left[t_{1}, \ldots, t_{n}\right]$, with each $t_{i}$ acting in degree -2 . If $\tau_{1}, \ldots, \tau_{n}$ is a basis of $D_{1}\left(A^{n}\right)$ dual to the basis $t_{1}, \ldots, t_{n}$, and if $\alpha=\left(\alpha_{1}, \ldots, \alpha_{n}\right)$ is a multi-index, we may write $\tau^{(\alpha)}=\tau_{1}^{\left(\alpha_{1}\right)} \cdots \tau_{n}^{\left(\alpha_{n}\right)}$ for the basis element dual to $t^{\alpha}=t_{1}^{\alpha_{1}} \ldots t_{n}^{\alpha_{n}} \in A\left[t_{1}, \ldots, t_{n}\right]$. With this notation,

$$
t_{i}\left(\tau^{(\alpha)}\right)=\tau_{1}^{\left(\alpha_{1}\right)} \cdots \tau_{i}^{\left(\alpha_{i}-1\right)} \cdots \tau_{n}^{\left(\alpha_{n}\right)},
$$

so that $t_{i}: D_{2 k+2}\left(A^{n}\right) \rightarrow D_{2 k}\left(A^{n}\right)$ is an epimorphism. We now consider the graded $A$-module $\tilde{\mathbf{F}}=D\left(A^{n}\right) \otimes \Lambda A^{m}$, and we define $\mathbf{F}$, as a graded $B$-module, to be $B \otimes \tilde{\mathbf{F}}=D\left(B^{n}\right) \otimes \Lambda B^{m}$. We define $\tilde{\partial}_{\mathbf{F}}: \tilde{\mathbf{F}} \rightarrow \tilde{\mathbf{F}}$ by

$$
\tilde{\partial}_{\mathbf{F}}=1 \otimes \partial+\sum_{i} t_{i} \otimes \sigma_{i}: D\left(A^{n}\right) \otimes \Lambda A^{m} \rightarrow D\left(A^{n}\right) \otimes \Lambda A^{m} .
$$

An easy calculation shows that

$$
\tilde{\partial}_{\mathbf{F}}^{2}=\sum_{i}\left(t_{i} \otimes 1\right) x_{i}
$$

so that $\left(B \otimes \tilde{\partial}_{\mathbf{F}}\right)^{2}=0$.

Thus with the differential $\partial_{\mathbf{F}}=B \otimes \tilde{\partial}_{\mathbf{F}}, \mathbf{F}$ is a minimal $B$-free complex; in fact $\mathbf{F}$ is the minimal $B$-free resolution of $K$. From (*), we see at once that $t_{i}\left(A,\left\{x_{i}\right\}, F\right)=$ $B \otimes\left(t_{i} \otimes 1\right)$; we will drop all the $\otimes$ signs, and simply write $t_{i}: \mathbf{F} \rightarrow \mathbf{F}$ for this map. We see that $t_{i} t_{j}=t_{j} t_{i}$, so $\mathbf{F}$ becomes a module over $B\left[t_{1}, \ldots, t_{n}\right]$.

The following consequence of the form of $\mathbf{F}$ will be useful to us later:

Proposition 2.1. Suppose that $A$ is a regular local ring, and that $x_{1}, \ldots, x_{n}$ are a maximal $A$-sequence, so that $B=A /\left(x_{1}, \ldots, x_{n}\right)$ is artinian. Let $\mathbf{F}$ be the minimal $B$-free resolution of the residue class field of $B$. Then the maps $t_{i}=t_{i}\left(A,\left\{x_{i}\right\}, F\right)$ : $\mathbf{F} \rightarrow \mathbf{F}$ can be chosen to commute with each other in such a way that $\mathbf{F}$ becomes an artinian $B\left[t_{1}, \ldots, t_{n}\right]$-module.

Proof. Suppose first that $x_{1}, \ldots, x_{n}$ are in the square of the maximal ideal $\mathscr{T}$, and let $y_{1}, \ldots, y_{n}$ be minimal generators for $\Re$. From the above construction, we see that $F=D\left(B^{n}\right) \otimes_{B} \Lambda B^{m}$ as a graded $B$-module, the action of $t_{j} \in$ $B\left[t_{1}, \ldots, t_{n}\right]$ being the natural action on the first factor. But 


$$
D\left(B^{n}\right)=\text { Hom }_{\text {graded } B \text {-modules }}\left(B\left[t_{1}, \ldots, t_{n}\right], B\right)_{\alpha}
$$

is artinian because it is the dual of a noetherian $B\left[t_{1}, \ldots, t_{n}\right]$-module. Since $\Lambda B^{m}$ is a finitely generated $B$-module, $\mathbf{F}$ is an artinian $B\left[t_{1}, \ldots, t_{n}\right]$-module, as claimed.

Now if not all the $x_{i}$ are contained in $\mathfrak{T}^{2}$, we may rearrange the $x_{i}$ so that $A^{\prime}=A /\left(x_{1}, \ldots, x_{r}\right)$ is a regular local ring with maximal ideal $\mathfrak{R}^{\prime}$, say, and the images $x_{i}^{\prime}$ of $x_{i}$ satisfy $x_{r+1}^{\prime}, \ldots, x_{n}^{\prime} \in \mathfrak{T}^{\prime 2}$. By the change of rings formula (Proposition 1.7), we may choose

$$
t_{i}\left(A,\left\{x_{i}\right\}, \mathbf{F}\right)=t_{i}\left(A^{\prime},\left\{x_{i}^{\prime}\right\}, \mathbf{F}\right) \text { for } i<r,
$$

reducing the proposition to the case just treated.

3. The main theorem. In this section we will prove the result which makes the maps $t_{i}$ useful in the analysis of free resolutions.

THEOREM 3.1. Let $A$ be a regular local ring whose residue class field is infinite, and let $I$ be an ideal of $A$ which can be generated by an $A$-sequence. Set $B=A / I$. If $\mathrm{F}: \ldots \rightarrow F_{1} \rightarrow F_{0}$ is the minimal $B$-free resolution of a finitely generated $B$-module then there exists an $A$-sequence $x_{1}, \ldots, x_{n}$ generating $I$ such that

$$
t_{1}\left(A,\left\{x_{j}\right\}, \mathbf{F}\right): F_{i+2} \rightarrow F_{i}
$$

is an epimorphism for sufficiently large $i$.

REMARKS. (1) If $t, t^{\prime}: \mathbf{F} \rightarrow \mathbf{F}$ are homotopic maps, of degree -2 , say, and if $t$ : $F_{i+2} \rightarrow F_{i}$ is onto, then so is $t^{\prime}: F_{i+2} \rightarrow F_{i}$, because $\mathbf{F}$ is minimal. Thus the statement of the theorem makes sense even though $t_{1}\left(A,\left\{x_{j}\right\}, F\right)$ is only defined up to homotopy.

(2) Theorem 3.1 is closely related to the main theorem (Theorem 3.1) of [G2]; in fact, given that the maps on Tor defined by Gulliksen are the same as those induced by the $t_{j}$, our Lemma 3.2 becomes a special case of Gulliksen's result. On the other hand, the direct way in which the $t_{j}$ are defined allows our proof to be somewhat simpler than that of [G2]. Theorem 3.1 of [G2] itself can be proved in the same style as our Lemma 3.2, using the construction of $\$ 7$ in place of that of $\$ 2$.

Problem. Is the restriction to the case of an infinite residue class field necessary?

The next example shows that no bound can be given for the values of $i$ for which $t_{1}: F_{i+2} \rightarrow F_{i}$ is not an epimorphism (but see Theorems 4.1 and 6.1).

EXAMPLe. Let $A$ be a regular local ring, and let $x_{1}, \ldots, x_{n}$ be a maximal $A$-sequence in the square of the maximal ideal of $A$, so that $B=A /\left(x_{1}, \ldots, x_{n}\right)$ is artinian. Suppose $n>2$. Let F: $\ldots \rightarrow F_{2} \rightarrow F_{1} \rightarrow F_{0}$ be the minimal $B$-free resolution of the residue class field $K$, as desribed in $\$ 2$. It is known [B] that $B$ is self-injective, so that

$$
\operatorname{Hom}(\mathrm{F}, B): F_{0}^{*} \rightarrow F_{1}^{*} \rightarrow \ldots
$$

is a minimal injective resolution of $\operatorname{Hom}(K, B)$. Moreover, $B$ has a unique minimal ideal, called the socle, which is principal; say socle $B=(s)$. The condition on $x_{1}, \ldots, x_{n}$ implies that $s$ is contained in the maximal ideal of $B$. We see that $\operatorname{Hom}(K, B) \cong(s) \cong K$. Identifying $F_{0}=R=F_{0}^{*}$, we get a doubly infinite minimal 
resolution

$$
\text { F }^{\text {Tate }}: \ldots F_{2} \rightarrow F_{1} \rightarrow R \stackrel{s}{\rightarrow} R \rightarrow F_{1}^{*} \rightarrow F_{2}^{*} \rightarrow \ldots
$$

Since rank $F_{i}<\operatorname{rank} F_{i+2}$, the maps

$$
t_{j}=t_{j}\left(A,\left\{x_{j}\right\}, \mathbf{F}^{\text {Tate }}\right): F_{i}^{*} \rightarrow F_{i+2}^{*}, \quad i>0,
$$

can never be epimorphisms.

Furthermore, the natural choice of the $t_{j}(\mathbf{F})$ makes

$$
t_{j}\left(F_{0}\right) \subset s F_{1}^{*} \text { and } t_{j}\left(F_{1}\right) \subset s R=s F_{0}^{*} \text {, }
$$

so that at this point, in the "middle" of $\mathbf{F}^{\text {Tate }}$, the maps $t_{j}$ are not even split. Thus if $N$ is any integer, then

$$
\mathrm{F}^{(-N)}: \ldots \rightarrow F_{N-1}^{*} \rightarrow F_{N}^{*}
$$

is a minimal free resolution for which the "sufficiently large" of Theorem 3.1 means "larger than $N$ ".

Proof of Theorem 3.1. Let $y_{1}, \ldots, y_{n}$ be any $A$-sequence generating $I$, and set $t_{j}=t_{j}\left(A,\left\{y_{j}\right\}, \mathbf{F}\right)$. We will show that there exist elements $a_{j} \in A$ such that the map

$$
t=t_{1}+\sum_{j=2}^{n} a_{j} t_{j}: F_{i+2} \rightarrow F_{i}
$$

is an epimorphism for large $i$.

Once this is done, we set

$$
x_{1}=y_{1}, \quad x_{j}=y_{j}-a_{j} y_{1}, \quad 2<j<n .
$$

Clearly $x_{1}, \ldots, x_{j}$ generate $I$. From Proposition 1.7 (applied with $A=A^{\prime}$ ), we see that

$$
t_{1}\left(A,\left\{x_{i}\right\}, \mathbf{F}\right)=t_{1}+\sum a_{i} t_{i}
$$

completing the proof.

Before choosing the $a_{j}$ we prove two lemmas. Recall that since the $t_{j}$ commute up to homotopy, they induce commutative maps on homology. In particular, if $M$ is the $B$-module resolved by $F$, and $K$ is the residue class field of $B$, the graded $B$-module $\operatorname{Tor}_{*}^{B}(M, K)$ becomes a $B\left[t_{1}, \ldots, t_{n}\right]$-module. The nontriviality of the $t_{i}$ is expressed by the next lemma:

LEMMA 3.2. $\operatorname{Tor}_{*}^{B}(M, K)$ is an artinian $B\left[t_{1}, \ldots, t_{n}\right]$-module.

Proof. Note that if $\mathbf{G}$ is the minimal $B$-free resolution of $K$, then by Proposition 1.6, the map induced on $\operatorname{Tor}_{*}^{B}(M, K)$ by $t_{j}\left(A,\left\{y_{j}\right\}, F\right)$ is the same as that induced by $t_{j}\left(A,\left\{y_{j}\right\}, \mathbf{G}\right)$.

To prove Lemma 3.2 , we will reduce to the case $\operatorname{dim} B=0$, and then apply Proposition 2.1.

To make the reduction, we wish to choose $d=\operatorname{dim} B$ elements $a_{1}, \ldots, a_{d} \in A$ such that $a_{1}, \ldots, a_{d}$ is a $B$-sequence and $A /\left(a_{1}, \ldots, a_{d}\right)$ is a regular local ring. By an easy inductive argument, it is enough to show the existence of an element $a_{1}$ in the maximal ideal $\mathfrak{N}$ of $A$ such that $a_{1}$ is a nonzero divisor on $B$ and $A /\left(a_{1}\right)$ is regular, supposing $d>1$. For the first of these conditions, it suffices that $a_{1}$ not be 
contained in $P_{1} \cup \ldots \cup P_{r}$ where $P_{1}, \ldots, P_{r}$ are the associated prime ideals of the ideal $I \subset A$. For the second, it suffices that $a_{1} \notin \Re^{2}$. However, since $I$ can be generated by an $A$-sequence, $\operatorname{depth} B=\operatorname{dim} B \geqslant 1$, so $\Re \neq P_{i}$ for all $i$. By a standard argument [Mat, Proposition 1.B], $\Re \nsubseteq \mathfrak{N}^{2} \cup P_{1} \cup \ldots \cup P_{r}$. Thus we can choose $a_{1} \in \mathscr{N}-\left(\mathscr{N}^{2} \cup P_{1} \cup \ldots \cup P_{r}\right)$.

Having chosen $a_{1}, \ldots, a_{d}$, we set $\bar{A}=A /\left(a_{1}, \ldots, a_{d}\right)$ and $\bar{B}=$ $B /\left(a_{1}, \ldots, a_{d}\right) B$. Let $N=\Omega_{d}(M)=$ image $F_{d} \rightarrow F_{d-1}$. Since $N$ is a $d$ th syzygy, $a_{1}, \ldots, a_{d}$ is an $N$-sequence. Since $K$ is annihilated by $a_{1}, \ldots, a_{d}$, we have

$$
\operatorname{Tor}_{i+d}^{B}(M, K)=\operatorname{Tor}_{i}^{B}(N, K)=\operatorname{Tor}_{i}^{\bar{B}}(\bar{B} \otimes N, K)
$$

for all $i \geqslant 1$. Since each $\operatorname{Tor}_{i}(M, K)$ is a finite-dimensional $K$-vectorspace, we see that it is enough to prove that $\operatorname{Tor}_{*}^{\bar{B}}(\bar{B} \otimes N, K)$ is an artinian $B\left[t_{1}, \ldots, t_{n}\right]$-module, where $t_{j}$ acts via the action of $\bar{B} \otimes t_{j}\left(A,\left\{y_{j}\right\}, \mathbf{F}\right)$ on $\bar{B} \otimes \mathbf{F}$, the $\bar{B}$-free resolution of $\bar{B} \otimes N$.

By Proposition 1.7 (with $A^{\prime}=\bar{A}$ ), we see that this action is the same as the action of $t_{j}\left(\bar{A},\left\{y_{j}\right\}, \bar{B} \otimes \mathbf{F}\right)$ (where we have written $y_{i}$ for the image of $y_{j}$ in $\left.\bar{B}\right)$. We thus see that it is enough to prove the lemma in the case $d=\operatorname{dim} B=0$.

We now consider $\operatorname{Tor}_{*}(M, K)=H_{*}(M \otimes G)$ as a subquotient of the $B\left[t_{1}, \ldots, t_{n}\right]$-module $M \otimes_{B} \mathbf{G}$. Since $M$ is a finitely generated $B$-module, and since $\mathbf{G}$ is an artinian $B\left[t_{1}, \ldots, t_{n}\right]$-module by Proposition 2.1 , we see that $\operatorname{Tor}_{*}(M, K)$ is artinian.

Note that since $\operatorname{Tor}_{*}^{B}(M, K)$ is annihilated by the maximal ideal $\mathscr{N}$ of $B$, it is even a $K\left[t_{1}, \ldots, t_{n}\right]$-module.

Before finishing the proof of Theorem 3.1, we need another lemma, which is in effect the dual of the standard result expressing the existence of "superficial" elements.

LEMMA 3.3. Let $K$ be an infinite field, and let $K\left[t_{1}, \ldots, t_{n}\right]$ be a graded polynomial ring, where the $t_{i}$ all have the same (negative) degree - e. If $T=\sum_{i>0} T_{i}$ is a graded artinian $K\left[t_{1}, \ldots, t_{n}\right]$-module, then there exist elements $\alpha_{j} \in K$ such that multiplication by the element $t=t_{1}+\sum_{j=2}^{n} \alpha_{j} t_{j}$ induces a surjective map $T_{i+e} \rightarrow T_{i}$ for all large $i$.

Proof. Let $T^{v}=\Sigma_{i>0} \operatorname{Hom}\left(T_{i}, K\right)=\operatorname{Hom}_{\text {graded } K \text {-modules }}(T, K)$. Each $t_{j}$ induces an operator on $T^{v}$ with degree $e>0$.

Since $T^{v}$ is the dual of an artinian module, $T^{v}$ is noetherian. In particular, the largest artinian submodule of $T^{v}$ has finite length, so that for a sufficiently large integer $N$, the truncation

$$
T^{v(N)}=\sum_{i>N} \operatorname{Hom}\left(T_{i}, K\right)
$$

contains no nonzero element annihilated by $\left(t_{1}, \ldots, t_{n}\right)$.

Let $P_{1}, \ldots, P_{r}$ be the associated primes of $0 \in T^{v(N)}$, so that $P_{1} \cup \ldots \cup P_{r}$ is the set of zero divisors on $T^{v(N)}$ [Mat, 7.B Corollary 2]. Since each $P_{i}$ annihilates some element of $T^{v(N)}$, no $P_{i}$ can contain the set $t_{1}+\sum_{i=2}^{n} K t_{i}$, which generates $\left(t_{1}, \ldots, t_{n}\right)$. But since $K$ is infinite, and since $t_{1}+\sum_{i=2}^{n} K t_{i}$ is the translation of a 
subspace of $K\left[t_{1}, \ldots, t_{n}\right]$, this implies that

$$
t_{1}+\sum_{i=2}^{n} K t_{i} \nsubseteq P_{1} \cup \cdots \cup P_{r},
$$

so there exists a linear form

$$
t=t_{1}+\sum_{j=2}^{n} \alpha_{j} t_{j} \quad\left(\alpha_{j} \in K\right)
$$

which is a nonzero divisor on $T^{v(N)}$. Dualizing again, we see that $t$ satisfies the conditions of the lemma.

Returning to the proof of the theorem, we apply Lemma 3.3 to the $K\left[t_{1}, \ldots, t_{n}\right]$-module $\operatorname{Tor}^{B}(M, K)$, obtaining a linear combination

$$
\bar{t}=t_{1}+\sum_{j=2}^{n} \alpha_{j} t_{j} \quad\left(\alpha_{j} \in K\right)
$$

of the $t_{i}$ such that $\bar{t}=\operatorname{Tor}_{i+2}(M, K) \rightarrow \operatorname{Tor}_{i}(M, K)$ is an epimorphism for all large $i$. Choose elements $a_{j} \in A$ such that the image of $a_{j}$ in $K$ is $\alpha_{j}$. Set $t=t_{1}+$ $\sum_{j=2}^{n} a_{j} t_{j}$. We claim that $t: F_{i+2} \rightarrow F_{i}$ is an epimorphism for all large $i$, which suffices to prove Theorem 3.1. By Nakayama's Lemma, it is enough to show that

$$
t \otimes K: F_{i+2} \otimes K \rightarrow F_{i} \otimes K
$$

is an epimorphism for large $i$.

But $t \otimes K=t_{1}+\sum_{j=2}^{n} \alpha_{j} t_{j}$, and because $\mathbf{F}$ is minimal, $\mathbf{F} \otimes K=\operatorname{Tor}_{*}^{B}(M, K)$, so the desired condition on $t \otimes K$ is the conclusion of Lemma 3.3.

4. Modules with bounded resolutions. As a first application of Theorem 3.1, we will analyse resolutions by free modules of bounded rank. This analysis will be carried further in the next two sections (see especially Theorem 5.2).

We will say that a complex $\mathbf{F}: \ldots \rightarrow F_{1} \rightarrow F_{0}$ is periodic of period 2 if there exists a map of complexes $s: \mathbf{F} \rightarrow \mathbf{F}$ of degree -2 such that $s: F_{i+2} \rightarrow F_{i}$ is an isomorphism for all $i \geqslant 0$.

THeOREM 4.1. Let $A$ be a regular local ring, and let $I \subset A$ be an ideal generated by an $A$-sequence. Set $B=A / I$, and let

$$
\text { F: } \ldots \rightarrow F_{1} \rightarrow F_{0}
$$

be a minimal $B$-free resolution. If $\left\{\right.$ rank $\left.F_{i}\right\}$ is bounded, then $\mathbf{F}$ becomes periodic of period 2 after at most $1+\operatorname{dim} B$ steps.

REMARK. (1) Using the ideas of Corollary 6.2, the result can be generalized to the case in which $B$ is an "abstract complete intersection"-that is, in which only the completion of $B$ is assumed to have the given form.

(2) We will see in Proposition 5.3 that $\operatorname{rank} F_{d+1}=\operatorname{rank} F_{d+2}=\ldots$, so " $\left\{\right.$ rank $\left.F_{i}\right\}$ bounded" implies " $\left\{\text { rank } F_{i}\right\}_{i>d+1}$ constant".

Proof. We first reduce to the case in which the residue class field of $A$ is infinite.

We must prove $\operatorname{Coker}\left(F_{d+2} \rightarrow F_{d+1}\right) \cong \operatorname{Coker}\left(F_{d+4} \rightarrow F_{d+3}\right)$. If such an isomorphism exists after a faithfully flat extension of $B$, then it exists already over $B$ [EGA, Chapter IV, Proposition 2.5.8]. On the other hand, writing $\mathfrak{N}$ for the 
maximal ideal of $A$, and letting $z$ be an indeterminate, $A[z]_{\mathscr{T}_{\mathcal{A}}[z]}$ is a faithfully flat extension of $A$, and

$$
A[z]_{\mathscr{R} A[z]} /\left(x_{1}, \ldots, x_{n}\right) A[z]_{\mathscr{R A} A z]} \cong B[z]_{\mathscr{T} B[z]}
$$

is a faithfully flat extension of $B$ which has an infinite residue class field. Thus we may assume from the outset that the residue class field of $A$ is infinite.

We can now apply Theorem 3.1, which shows that if $x_{1}, \ldots, x_{n}$ is a well-chosen set of generators for $\left(x_{1}, \ldots, x_{n}\right)$, then there exists an integer $N$ such that $t_{1}=t_{1}\left(A,\left\{x_{i}\right\}, \mathbf{F}\right): F_{i+2} \rightarrow F_{i}$ is an epimorphism for all $i>N$.

From this property of $t_{1}$ it follows that $\operatorname{rank} F_{N}<\operatorname{rank} F_{N+2}<\ldots$ and rank $F_{N+1} \leqslant$ rank $F_{N+3} \leqslant \ldots$ Since rank $F_{i}$ \} is bounded, both these increasing sequences become stationary; as soon as this happens $t_{1}$ becomes an isomorphism. Thus there exists an integer $M$ such that $t_{1}: F_{i+2} \rightarrow F_{i}$ is an isomorphism for $i>M$. Thus $\mathbf{F}$ becomes periodic after $M$ steps.

It remains to show that we can take $M=d+1$. To do this we reduce to the case $\operatorname{dim} B=0$ : To this end, let $x_{n+1}, \ldots, x_{n+d} \in A$ be a $B$-sequence and let $\bar{B}=$ $B /\left(x_{n+1}, \ldots, x_{n+d}\right)$. For any $i, t_{1}: F_{i+2} \rightarrow F_{i}$ is an isomorphism if $\bar{B} \otimes t_{1}: \bar{B} \otimes$ $F_{i+2} \rightarrow \bar{B} \otimes F_{i}$ is an isomorphism. Furthermore, by Lemma 0.1(i)

$$
\bar{B} \otimes F^{d}: \ldots \rightarrow \bar{B} \otimes F_{d+1} \rightarrow \bar{B} \otimes F_{d}
$$

is a minimal free resolution over $\bar{B}$. Thus we may assume that $\operatorname{dim} B=d=0$, and prove that $t_{1}: F_{i+2} \rightarrow F_{i}$ is an isomorphism for all $i>1$. To do this we will use the self-injectivity of $B[B]$ which implies that any truncation of $F$ uniquely determines all but $F_{0}$ :

LEMMA 4.2. Let $B$ be a zero-dimensional Gorenstein local ring. If

$$
\mathrm{F}: \ldots \rightarrow F_{2} \rightarrow F_{1} \rightarrow F_{0}
$$

is a minimal $B$-free resolution, then for each $i>1, F_{i}$ is the injective envelope of Coker $F_{i+2} \rightarrow F_{i+1}$.

Proof. Let $C_{i}=\operatorname{Coker}\left(F_{i+2} \rightarrow F_{i+1}\right)$. Since $B$ is self-injective, and $\mathbf{F}$ is minimal and exact, the free complex $\operatorname{Hom}(\mathbf{F}, B)$ is also minimal and exact. Thus if $i \geqslant 1$,

$$
\operatorname{Hom}\left(F_{i-1}, B\right) \rightarrow \operatorname{Hom}\left(F_{i}, B\right) \rightarrow \operatorname{Hom}\left(C_{i}, B\right) \rightarrow 0
$$

is the beginning of a minimal free resolution. But the functor $\operatorname{Hom}(, B)$ is a duality on the category of finitely generated $B$-modules. Thus $0 \rightarrow C_{i} \rightarrow F_{i} \rightarrow F_{i-1}$ is the beginning of a minimal injective resolution of $C$; in particular $F_{i}$ is the injective envelope of $C_{i}$.

Returning to the proof of Theorem 4.1, we may assume by induction that $t_{1}$ : $F_{i+2} \rightarrow F_{i}$ is an isomorphism for all $i>2$, so in particular

$$
\operatorname{Coker}\left(F_{5} \rightarrow F_{4}\right) \cong \operatorname{Coker}\left(F_{3} \rightarrow F_{2}\right) \text {. }
$$

By Lemma 4.2, there exists an isomorphism $s$ making the diagram

$$
\begin{array}{lllll}
F_{5} & \rightarrow & F_{4} & \rightarrow & F_{3} \\
t_{1} \downarrow & & t_{1} \downarrow & & \downarrow s \\
F_{3} & \rightarrow & F_{2} & \rightarrow & F_{1}
\end{array}
$$


commute. If we regard (*) as a map of complexes, then we see by Proposition 1.3, that the diagram

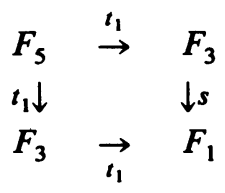

commutes up to homotopy. Since $F_{3} \stackrel{s t_{1}^{-1}}{\rightarrow} F_{1}$ is an isomorphism and $\mathrm{F}$ is minimal, $t_{1}$ : $F_{3} \rightarrow F_{1}$ is an isomorphism as well.

5. Resolutions of period 2. Motivated by Theorem 4.1, and by the fact (to be proved in the next section) that over a hypersurface every minimal free resolution becomes periodic of period 2, we take up a general study of resolutions of period 2 .

We begin with a general construction of periodic resolutions. It will turn out (Theorem 5.2) that all periodic resolutions over complete intersections arise from this construction.

A matrix factorization of an element $x$ in a ring $A$ is an ordered pair of maps of free $A$-modules $(\varphi: F \rightarrow G, \Psi: G \rightarrow F)$ such that $\varphi \Psi=x \cdot 1_{G}$ and $\Psi \varphi=x \cdot 1_{F}$.

Note that if $(\varphi, \Psi)$ is a matrix factorization of $x$, then $x$ annihilates Coker $\varphi$.

Proposition 5.1. Let $(\varphi: F \rightarrow G, \Psi: G \rightarrow F)$ be a matrix factorization of an element $x$ in a ring $A$. Set $B=A /(x)$, and write - for reduction modulo $x$. If $(x) /\left(x^{2}\right)$ is free over $B$, then the periodic complex

$$
\mathbf{F}(\varphi, \Psi): \ldots \rightarrow \bar{F} \stackrel{\bar{\Phi}}{\rightarrow} \bar{G} \stackrel{\bar{\Psi}}{\rightarrow} \bar{F} \stackrel{\bar{\Phi}}{\rightarrow} \bar{G}
$$

is a $B$-free resolution of Coker $\varphi$, and $t(x, F(\varphi, \Psi))$ is the identity.

Proof. Since $x($ Coker $\varphi)=0$, we have Coker $\varphi=\operatorname{Coker} \bar{\varphi}$. The relation of $\varphi$ and $\Psi$ being symmetric, it will be enough to show the exactness at $\bar{F}$. If $K$ is a free $A$-module and $\kappa: K \rightarrow F$ is a map such that $\bar{\varphi} \bar{\kappa}=0$, we must show that $\bar{\kappa}$ factors through $\bar{\Psi}$.

Since $\bar{\varphi} \bar{\kappa}=(\overline{\varphi \kappa})$ there exists a map $\gamma: K \rightarrow G$ such that $\varphi \kappa=x \gamma$. Thus

$$
x \kappa=\Psi \varphi \kappa=\Psi(x \gamma)=x \Psi \gamma .
$$

Since $(x) /\left(x^{2}\right)$ is free over $A /(x)$, this implies $\bar{\kappa}=(\overline{\Psi \gamma})=\bar{\Psi} \bar{\gamma}$, as required.

To prove that $t(A, x, \mathbf{F}(\varphi, \Psi))$ is the identity, consider the obvious lifting

$$
\tilde{\mathbf{F}}(\varphi, \Psi): \ldots \rightarrow \underset{\rightarrow}{\rightarrow} G \stackrel{\Psi}{\rightarrow} F \stackrel{\varphi}{\rightarrow} G .
$$

Since $(\varphi, \Psi)$ is a matrix factorization, $\tilde{t}: F \rightarrow F$ and $\tilde{t}: G \rightarrow G$ may be taken to be the identity maps.

THEOREM 5.2. Let $A$ be a regular local ring with infinite residue class field, and let $I \subset A$ be an ideal generated by an $A$-sequence. Set $B=A / I$. If $F$ is a periodic minimal $B$-free resolution, then there exist a local ring $B_{1}$, a nonzero divisor $x \in B_{1}$, and a matrix factorization $(\varphi, \Psi)$ of $x$ over $B_{1}$, such that $\mathbf{F} \simeq \mathbf{F}(\varphi, \Psi)$. 
Proof. As in the proof of Theorem 3.1, a set of generators $x_{1}, \ldots, x_{n}$ of $I$ can be chosen such that $t=t_{1}\left(A,\left\{x_{i}\right\}, \mathbf{F}\right): F_{i+2} \rightarrow F_{i}$ is an isomorphism for all $i \geqslant 0$ (because $\mathbf{F}$ is periodic, we do not need to truncate $\mathbf{F})$. Set $B_{1}=A /\left(x_{2}, \ldots, x_{n}\right)$, and let $x$ be the image of $x_{1}$ in $B_{1}$. From Proposition 1.7, we see that $t=$ $t\left(B_{1}, x, \mathbf{F}\right)$. Let $\tilde{\mathbf{F}}: \rightarrow \tilde{F}_{2} \stackrel{\Psi}{\rightarrow} \tilde{F}_{1} \stackrel{\varphi}{\rightarrow} \tilde{F}_{0}$ be the sequence of maps of $B_{1}$-free modules used to define $t$, and let $\tilde{t}=\tilde{t}\left(B_{1}, x, \tilde{F}\right)$. By Nakayama's Lemma, $\tilde{t}$ is an isomorphism. Identifying $\tilde{F}_{0}$ with $\tilde{F}_{2}$ and $\tilde{F}_{1}$ with $\tilde{F}_{3}$ by means of $\tilde{t}$, we see that $(\varphi, \Psi)$ is a matrix factorization of $x$. Since $F(\varphi, \Psi)$ and $F$ are both minimal free resolutions of Coker $(B \otimes \varphi)$, they are isomorphic.

Proposition 5.3. Let $R$ be a noetherian ring, and let $\ldots \stackrel{\varphi}{\rightarrow} G \stackrel{\Psi}{\rightarrow} F \stackrel{\varphi}{\rightarrow} G$ be a free resolution of a finitely generated $R$-module which is periodic of period 2 . Then rank $F=\operatorname{rank} G$.

Proof. We may suppose that $\mathrm{F}$ has the form $\mathrm{F}: \ldots \rightarrow F \stackrel{\varphi}{\rightarrow} G \stackrel{\boldsymbol{\Psi}}{\rightarrow} F \stackrel{\varphi}{\rightarrow} G \stackrel{\Psi}{\rightarrow} \ldots$ By localizing at a minimal prime of $B$ (which does not affect the rank of $F$ or of $G$ ) we may assume that $B$ is artinian, and thus of finite length. Now

$$
\text { length } F=\text { length(image } \varphi)+ \text { length(image } \Psi)=\text { length } G
$$

so

$$
\text { rank } \begin{aligned}
F & =\text { length } F / \text { length } B \\
& =\text { length } G / \text { length } B=\operatorname{rank} G .
\end{aligned}
$$

COROllary 5.4. If ( $\varphi: F \rightarrow G, \Psi: G \rightarrow F)$ is a matrix factorization of an element $x \in A$ such that $(x) /\left(x^{2}\right)$, if free over $A /(x)$, then $\operatorname{rank} F=\operatorname{rank} G$.

Proof. Apply Proposition 5.1 and Proposition 5.3.

If $x$ is a nonzero divisor, more can be said. We will use the following definitions: Let $\varphi: F \rightarrow G$ be a map of free modules, with rank $G=g$. We write Fit ${ }_{k} \varphi$ for the ideal generated by the minors of $\varphi$ of order $g-k$. (Fit $\boldsymbol{F} \varphi$ is the " $k$ th Fitting invariant" of Coker $\varphi$ [Kap]). If $F$ and $G$ have the same rank, then $F_{i t} \varphi$ is generated by the determinant of any matrix representing $\varphi$, and we write $\mathrm{Fit}_{0} \varphi=$ $(\operatorname{det} \varphi)$.

Proposition 5.5. Let $x \in A$ be a nonzero divisor, and let $\varphi: F \rightarrow G$ be a map between free modules. There exists a matrix factorization of the form $(\varphi, \Psi)$ if and only if

(a) rank $F=\operatorname{rank} G$,

(b) det $\varphi$ is a nonzero divisor, and

(c) $x \cdot \operatorname{Fit}_{1}(\varphi) \subset(\operatorname{det} \varphi)$.

If $\Psi$ exists, it is uniquely determined by $\varphi$.

Proof. Suppose $(\varphi, \Psi)$ is a matrix factorization of $x$. Condition (a) follows from Corollary 5.4. Since $\Psi \varphi=x \cdot 1_{F}$ is a monomorphism, $\varphi$ must also be a monomorphism, and (b) follows ("Mc Coy's Theorem": see [Kap]). Choosing bases for $F$ and $G$, and regarding $\varphi$ as a square matrix, we let $\varphi^{c}$ be the matrix of cofactors of $\varphi$, so that $\varphi^{c} \varphi=\varphi \varphi^{c}=(\operatorname{det} \varphi) \cdot 1$. The entries of $\varphi^{c}$ are precisely the generators of 
Fit $_{1}(\varphi)$. But $\varphi \Psi=x \cdot 1_{G}$, so

$$
x \varphi^{c}=\Psi \varphi \varphi^{c}=\Psi \cdot(\operatorname{det} \varphi),
$$

proving (c). From this last equation, we see that $\Psi=x \varphi^{c} /$ det $\varphi$ (over the ring $\left.A\left[(\operatorname{det} \varphi)^{-1}\right]\right)$. Since $\operatorname{det} \varphi$ is a nonzero divisor, this shows that $\Psi$ is determined by $\varphi$.

Conversely, if (a) and (c) are satisfied, then there exists a matrix $\Psi$ such that $x \cdot \varphi^{c}=\Psi(\operatorname{det} \varphi)$. Thus $\operatorname{det} \varphi \cdot \Psi \varphi=x \varphi^{c} \varphi=x \cdot \operatorname{det} \varphi$, and $(\operatorname{det} \varphi) \varphi \Psi=x \varphi \varphi^{c}=$ $x \cdot \operatorname{det} \varphi$. If now (b) is satisfied, $\varphi \Psi=x=\Psi \varphi$ as required.

Remark. (1) Let $x \in A$ be a nonzero divisor, and suppose $\varphi: F \rightarrow G$ and $\Psi$ : $G \rightarrow F$ are maps of free modules with rank $F=\operatorname{rank} G$. Then $\varphi \Psi=x \cdot 1_{G}$ implies that $\Psi=x \varphi^{-1}$ over the ring $A\left[x^{-1}\right]$, so $\Psi \varphi=x \cdot 1_{G}$. Thus half the condition for $(\varphi, \Psi)$ to be a matrix factorization implies the other half.

(2) If $\varphi: F \rightarrow G$ is a map between free modules of the same rank such that det $\varphi$ is a nonzero divisor, then $\operatorname{ann}_{R}(\operatorname{Coker} \varphi)=\left(\right.$ Fit $_{1}(\varphi)$ : det $\left.\varphi\right)($ see, for example, [B-E 3]). Thus condition (c) is equivalent to $x \operatorname{Coker} \varphi=0$.

We now consider the case in which $x$ is a prime nonzero divisor, so that $B=A /(x)$ is an integral domain. Let $Q$ be the quotient field of $B$. If $M$ is a $B$-module, we define $\operatorname{rank}_{B} M$ to be the dimension of the $Q$-vectorspace $Q \otimes M$.

Proposition 5.6. Suppose that $x \in A$ is a prime nonzero divisor, and that $(\varphi, \Psi)$ is a matrix factorization of $x$. Write $\operatorname{det} \varphi=x^{k} u$, with $u \notin(x)$. Then $\operatorname{rank}_{B}($ Coker $\varphi)$ $=k$.

Proof. Localizing at $(x)$, we may assume that $A$ is a discrete valuation ring with parameter $x$. It follows easily (for example from the theory of elementary divisors) that $(\varphi, \Psi) \cong\left(1_{g-k} \oplus x 1_{k}, x 1_{g-k} \oplus 1_{k}\right)$, where $g=$ rank $G$. The desired result follows.

Finally, if ( $\varphi: F \rightarrow G, \Psi: G \rightarrow F$ ) is a matrix factorization of $x$, consider the problem of explicitly determining $\Psi$ from $\varphi$. If $x$ is a prime nonzero divisor, then, since $(\operatorname{det} \varphi)(\operatorname{det} \Psi)=x^{g}$, where $g=\operatorname{rank} G$, we must have $\operatorname{det} \varphi=x^{k} u$, for some unit $u$. If $k=1$, then letting $\varphi^{c}$ be the matrix of cofactors of $\varphi,\left(\varphi, u^{-1} \varphi^{c}\right)$ is also a matrix factorization of $x$, so by Proposition 5.5, $\Psi=u^{-1} \varphi^{c}$. The following proposition generalizes this remark. Recall that if $F$ and $G$ have rank $g$, then a choice of generators in $\wedge^{8} F$ and $\wedge^{8} G$ induces isomorphisms

$$
\stackrel{k}{\wedge} \stackrel{\delta}{\rightarrow}_{\rightarrow}^{g-k} \wedge^{*} F^{*}, \quad \stackrel{k}{\wedge} G \stackrel{\delta}{\rightarrow} \stackrel{g-k}{\wedge} G^{*}
$$

With this notation, $\varphi^{c}=\delta^{-1}\left(\bigwedge^{g^{-1}} \varphi^{*}\right) \delta$. More generally [Bou, Chapitre III]

$$
\bigwedge^{k} \varphi \cdot \delta^{-1}\left(\stackrel{g-1}{\wedge}^{*} \varphi^{*}\right) \delta=\stackrel{g}{\wedge} \varphi
$$

Proposition 5.7. Suppose $(\varphi, \Psi)$ is a matrix factorization of a nonzero divisor $x \in A$, and that $\operatorname{det} \varphi=x^{k} u$, for some $u \in A$. Then

$$
u \wedge \Psi=\delta^{-1}\left(\wedge^{g-k} \varphi^{*}\right) \delta
$$


Proof. Both $\left(\wedge^{k} \varphi, u \wedge^{k} \Psi\right)$ and $\left(\wedge^{k} \varphi, \delta^{-1} \wedge^{8-k} \varphi^{*} \delta\right)$ are matrix factorizations of $x u=\operatorname{det} \varphi$, which is a nonzero divisor by Proposition 5.5, condition (b). The conclusion follows from the last statement of Proposition 5.5.

6. Resolutions and Cohen-Macaulay modules over a hypersurface. In this section, we will show that minimal resolutions over a hypersurface are eventually periodic (Theorem 6.1), and deduce some consequences for these rings. Theorem 6.1 could be deduced from the machinery we have already built up; but we have chosen to give an elementary proof, which foreshadows some of the ideas in the next two sections. We are grateful to $\mathbf{M}$. Hochster for a remark which simplified our original proof of Theorem 6.1.

We will say that a complex F: $\ldots \rightarrow F_{1} \rightarrow F_{0}$ becomes periodic of period 2 after $k$ steps if the truncation $\mathbf{F}^{(k)}: \ldots \rightarrow F_{k+1} \rightarrow F_{k}$ is periodic of period 2 .

If $B$ is a local ring, then a (finitely generated) $B$-module $M$ is a maximal Cohen-Macaulay module if $\operatorname{depth} M=\operatorname{dim} B$ (the largest possible value); that is, if there is a system of parameters $p_{1}, \ldots, p_{d}$ in $B$ which form an $M$-sequence.

Theorem 6.1. Let $A$ be a regular local ring, $x \in A$, and let $B=A / x$. Let $d=\operatorname{dim} A$. If

$$
\mathrm{F}: \ldots \rightarrow F_{1} \rightarrow F_{0}
$$

is the minimal $B$-free resolution of a finitely generated $B$-module $M$, then:

(i) F becomes periodic of period 2 after $d+1$ steps,

(ii) $\mathbf{F}$ is periodic (necessarily of period 2) if and only if $M$ is a maximal Cohen-Macaulay $B$-module with no free summand.

(iii) Every periodic minimal free resolution over $B$ has the form $F(\varphi, \Psi)$ for some matrix factorization $\varphi, \Psi$ of $x$ over $A$. In particular, the map $t(A, x, F)$ gives the periodicity.

The following corollary generalizes the Auslander-Buchsbaum-Serre characterization of regular local rings as those of finite global dimension:

COROLlARY 6.2. Let $R$ be a local ring. The following conditions are equivalent:

(i) The maximal ideal of $R$ can be generated by $1+\operatorname{dim} R$ elements, and the zero ideal of $R$ is analytically unmixed (that is, 0 is unmixed in the completion of $R$ ).

(ii) The minimal free resolution of any finitely generated $R$-module becomes periodic, of period 2, after $1+\operatorname{dim} R$ steps.

(iii) There exists a free resolution $\mathbf{F}: \ldots \rightarrow F_{1} \rightarrow F_{0}$ of the residue class field of $R$ such that for some $n$, rank $F_{n}<n$.

REMARK. A ring satisfying condition (i) is sometimes called an "abstract hypersurface", since its completion can be written as a regular local ring modulo a principal ideal. Since condition (ii) of the corollary is preserved by localization, one consequence of the corollary is that any localization of an abstract hypersurface is an abstract hypersurface. Using the material of $\$ 8$, an analogous result could be proved for "abstract complete intersections" of codimension $<r$ for any $r$. 
Proof of Theorem 6.1. By Lemma 0.1, part (i) follows from part (ii). For the "only if" part of (ii), note that if $M$ has a periodic minimal free resolution $\ldots \rightarrow G \rightarrow F \rightarrow G \rightarrow M \rightarrow 0$, then $M \cong \operatorname{ker}(G \rightarrow F)$, so Lemma 0.1 applies to show that $M$ is a maximal Cohen-Macaulay module with no free summands.

We will now show that if $M$ is a maximal Cohen-Macaulay $B$-module without free summands, then the minimal $B$-free resolution of $M$ has the form $F(\varphi, \Psi)$, where $(\varphi, \Psi)$ is a matrix factorization of $x$. By virtue of Proposition 5.1 this will conclude the proof.

By the theorem of Auslander-Buchsbaum-Serre,

$$
\text { projective dimension }_{A}(M)=\operatorname{dim} A-\operatorname{depth} M=1 .
$$

Let $\mathrm{F}^{\#}: 0 \rightarrow F \stackrel{\varphi}{\rightarrow} G \rightarrow M \rightarrow 0$ be the minimal free resolution of $M$ as an $A$-module. Since $M$ is annihilated by $x, x G \subset$ (image $\varphi$ ), so there exists a map $\Psi: G \rightarrow F$ so that $\varphi \Psi=x 1_{G}$. Multiplying by $\varphi$ on the right we get $\varphi \Psi \varphi=x \varphi$. Since $\varphi$ is a monomorphism, this implies $\Psi \varphi=x \cdot 1_{F}$, so $(\varphi, \Psi)$ is a matrix factorization of $x$ over $A$.

Writing - for reduction modulo $x$,

$$
\mathbf{F}(\varphi, \Psi): \ldots \stackrel{\bar{\Phi}}{\rightarrow} \bar{G} \stackrel{\bar{\Psi}}{\rightarrow} \bar{F} \stackrel{\bar{\Phi}}{\rightarrow} \bar{G}
$$

is a periodic free resolution of $M$ by Proposition 5.1. It remains to show that $\mathrm{F}(\varphi, \Psi)$ is minimal. Since $0 \rightarrow F \stackrel{\varphi}{\rightarrow} G$ was chosen to be minimal, we need only show that if $\Re$ is the maximal ideal of $B$, then $\bar{\Psi}(\bar{G}) \subset \mathfrak{T} \bar{F}$. However, since $\mathbf{F}(\varphi, \Psi)$ is exact,

$$
\bar{\Psi}(\bar{G}) \cong \operatorname{Coker} \bar{\varphi} \cong \bar{M} \cong M .
$$

If $\bar{\Psi}(\bar{G})$ were not contained in $\Re \bar{F}$, then $\bar{\Psi}(\bar{G})$ would contain a basis element of $\bar{F}$, so $\bar{\Psi}(\bar{G})$ would have a $B$-free summand. Since we assumed that $M$ had no free summands, this concludes the proof.

Proof of Corollary 6.2. (i) $\Rightarrow$ (ii). Let F: $\ldots \rightarrow F_{2} \rightarrow F_{1} \rightarrow F_{0}$ be the minimal free resolution of an $R$-module $M$. Because $F$ is minimal, it suffices to prove that $\Omega_{i+2}^{R}(M) \cong \Omega_{i}^{R}(M)$ for large $i$. But it follows from Cohen's structure theorems that the completion $\hat{R}$ can be written in the form $\hat{R}=A / I$, where $A$ is a regular local ring, whose dimension is the number of generators $1+\operatorname{dim} R$ of the maximal ideal of $R$, and $I$ is an ideal of $A$. The hypothesis (i) on $R$ means that the associated primes of 0 in $\hat{R}$ all have the same dimension, so $I$ is unmixed of height 1 . Since $A$ is regular, $A$ factorial, so $I$ is principal, say $I=(x)$.

By Theorem 3.1(i), the minimal resolution $\hat{R} \otimes \mathrm{F}$ of $\hat{R} \otimes M$ becomes periodic of period 2. Writing $\Omega_{k}^{R}(M)$ for the $k$ th-syzygy module of $M$ as an $R$-module, and using the corresponding notation for syzygies over $\hat{R}$, we have:

$$
\hat{R} \otimes \Omega_{i+2}^{R}(M) \cong \Omega_{i+2}^{\hat{R}}(\hat{R} \otimes M) \cong \Omega_{i}^{\hat{R}}(\hat{R} \otimes M) \cong \hat{R} \otimes \Omega_{i}^{R}(M)
$$

for $i$ large. 
The fact that $\hat{R} \otimes \Omega_{i+2}^{R}(M) \cong \hat{R} \otimes \Omega_{i}^{R}(M)$ implies that $\Omega_{i+2}^{R}(M) \cong \Omega_{i}^{R}(M)$ [EGA, Chapitre IV, 2.5.8]. (SKETCH OF PROOF. The maps

$$
\hat{R} \otimes \Omega_{i+2}^{R}(M) \underset{{ }_{\beta}}{\stackrel{\alpha}{R}} \hat{R} \otimes \Omega_{i}^{R}(M)
$$

that give the isomorphism may be approximated $\mathfrak{T}$-adically by maps of the form $\hat{R} \otimes \alpha^{\prime}$ and $\hat{R} \otimes \beta^{\prime}$. For sufficiently good approximations, $\alpha^{\prime}$ and $\beta^{\prime}$ will be onto. But then $\alpha^{\prime} \beta^{\prime}$ and $\beta^{\prime} \alpha^{\prime}$, being epimorphisms from a noetherian module to itself, are isomorphisms.) This proves (i) $\Rightarrow$ (ii).

(ii) $\Rightarrow$ (iii) is obvious.

(iii) $\Rightarrow$ (i). Condition (i) is really a condition on the completion of $R$, and condition (iii) passes to the completion, so we may assume that $R$ is complete. Write $R=S / I$, where $S$ is a regular local ring of the minimal possible dimension; say $\operatorname{dim} S=d$. The number $d$ will also be the minimal number of generators of the maximal ideal of $R$. Suppose $I$ is minimally generated by $e$ elements. Then by [G1], the underlying graded $R$-module of $F$ may be written as a tensor product of which one of the factors is $D\left(R^{e}\right) \otimes \Lambda R^{d}$, where $\Lambda R^{d}$ is the exterior algebra on $d$ generators of degree 1 and $D\left(R^{e}\right)$ is the divided power algebra on $e$ elements of degree 2. (See $\$ 2$ or [G-L] for more details.)

If $e=0$, then $R=S$ is regular, and (i) is trivial. If $e=1$, then by the unmixedness theorem [Mat, Theorem 16.D], $I$ is unmixed of height 1 , and (i) follows. If $d \leqslant 1$, then $I$ is principal, so $e=1$. Thus we are done unless $d>2, e>2$. But then a straightforward computation shows that

$$
\begin{aligned}
\operatorname{rank} F_{k} & \geqslant \operatorname{rank}\left(D\left(R^{e}\right) \otimes \Lambda R^{d}\right)_{k} \\
& \geqslant \operatorname{rank}\left(D\left(R^{2}\right) \otimes \Lambda R^{2}\right)_{k}=k,
\end{aligned}
$$

contradicting condition (iii).

As a consequence of Theorems 6.1 and 5.2, we see that periodic free resolutions and Cohen-Macaulay modules over a hypersurface correspond to matrix factorizations. To be more precise, we introduce some terminology:

Two matrix factorizations $(\varphi: F \rightarrow G, \Psi: G \rightarrow F)$ and $\left(\varphi^{\prime}: F^{\prime} \rightarrow G^{\prime}, \Psi^{\prime}: G^{\prime} \rightarrow\right.$ $\left.F^{\prime}\right)$ of $x \in A$ are equivalent if there exist isomorphisms $\alpha, \beta$ making the diagram

$$
\begin{array}{lllll}
F & \stackrel{\varphi}{\rightarrow} & G & \stackrel{\Psi}{\rightarrow} & F \\
\alpha \downarrow & & \beta \downarrow & & \alpha \downarrow \\
F^{\prime} & \overrightarrow{\varphi^{\prime}} & G^{\prime} & \overrightarrow{\Psi^{\prime}} & F^{\prime}
\end{array}
$$

commute. (If $x$ is a nonzero divisor, we need only require that one square commutes.) The direct sum of $(\varphi, \Psi)$ and $\left(\varphi^{\prime}, \Psi^{\prime}\right)$ is $\left(\varphi \oplus \varphi^{\prime}, \Psi \oplus \Psi^{\prime}\right)$. The trivial factorizations are $(1, x)$ and $(x, 1)$. A factorization is reduced if it is not equivalent to a factorization having a trivial factorization as a direct summand. If $x$ is a nonzero divisor and $A$ is local with maximal ideal $\mathscr{N}$, it is easy to show that $(\varphi, \Psi)$ is reduced if and only if $\varphi(F) \subset \Re(G$ and $\Psi(G) \subset \mathscr{R} F$. 
COROllary 6.3. Let $A$ be a regular local ring, and let $B=A /(x)$ be a proper factor ring. Write ${ }^{-}$for reduction modulo $x$. The associations

$$
(\varphi, \Psi) \mapsto \mathrm{F}_{(\varphi, \Psi)}: \ldots \stackrel{\bar{\varphi}}{\rightarrow} \bar{G} \stackrel{\bar{\Psi}}{\rightarrow} \bar{F} \stackrel{\bar{\varphi}}{\rightarrow} \bar{G}
$$

and

$$
(\varphi, \psi) \mapsto M_{(\varphi, \Psi)}=\operatorname{Coker} \varphi
$$

induce bijections between the set of

(1) Equivalence classes of reduced matrix factorizations of $x$ over $A$.

(2) Isomorphism classes of nontrivial periodic minimal free resolutions over $B$.

(3) Maximal Cohen-Macaulay B-modules without free summands.

If $x \in A$ is a prime, we can be still more explicit:

COROLlaRy 6.4. Let $A$ be a regular local ring with maximal ideal $\Re$ and let $F$ be a free $A$-module of rank $n$. Let $x \in A$ be a prime element and set $B=A /(x)$. There is a bijection between the sets

(i) Maximal Cohen-Macaulay $B$-modules of rank $k$ requiring exactly $n$ generators.

(ii) Equivalence classes of endomorphisms $\varphi: F \rightarrow F$ with $\varphi(F) \subset \Re F$, det $\varphi=$ $x^{k}$, and $\mathrm{Fit}_{1}(\varphi) \subset\left(x^{k-1}\right)$. (The equivalence relation is given by $\varphi \sim P \varphi Q$ if $P$ and $Q$ are automorphisms of $F$ with $\operatorname{det} P \operatorname{det} Q=1$.)

The bijection is induced by $\varphi \mapsto$ Coker $\varphi$.

REMARK. If $k=1$, the condition Fit $\varphi \subset\left(x^{k-1}\right)$ becomes vacuous.

Suppose $\varphi$ satisfies (ii). From Proposition 5.5, we see that there is a matrix factorization of $x$ of the form $(\varphi, \Psi)$. (In particular, Coker $\varphi$ is a $B$-module!) Corollary 6.4 now follows from Corollary 6.3 and Proposition 5.6.

7. Construction of free resolutions over a complete intersection. In this section we will give a construction for free resolutions over a comp!ete intersection which generalizes the constructions of $\$ \S 2$ and 5 (and also the construction given in [G2]). Its disadvantage is that it does not always produce minimal resolutions. We will see in the next section that this can be overcome "in relative codimension 1".

Let $A$ be a ring, let $x_{1}, \ldots, x_{n}$ be an $A$-sequence, and set $B=A /\left(x_{1}, \ldots, x_{n}\right)$. Given any $A$-free resolution of a $B$-module $M$, and certain additional data, we will construct a $B$-free resolution of $M$, and we will describe the action of the $t_{i}\left(A,\left\{x_{i}\right\},-\right)$ on this resolution.

Let $\mathrm{F}: \ldots \stackrel{\partial}{\rightarrow} F_{i+1} \stackrel{\partial}{\rightarrow} F_{i} \stackrel{\partial}{\rightarrow}$ be a complex of $A$-modules. We will distinguish between endomorphisms of degree $k$ of $\mathbf{F}$ as a graded module (that is, a family of maps $s: F_{i} \rightarrow F_{i+k}$, for all $i$ ) and endomorphisms of degree $k$ of $\mathbf{F}$ as a complex ( $s$ as above, subject to $s \partial=\partial s$ ).

We also introduce some multi-index conventions. A multi-index (of length $n$ ) is a sequence $\alpha=\left\langle\alpha_{1}, \ldots, \alpha_{n}\right\rangle$ where each $\alpha_{i}$ is an integer $\geqslant 0$. We write $0=$ $\langle 0, \ldots, 0\rangle$. The order of a multi-index $\alpha$ is

$$
|\alpha|=\sum_{i=1}^{n} \alpha_{i}
$$


The sum $\alpha+\beta$ is defined as $\left\langle\alpha_{1}+\beta_{1}, \ldots, \alpha_{n}+\beta_{n}\right\rangle$.

If $\mathrm{F}$ is an $A$-free resolution (with $A$ as above), we will show how to make $B \otimes D\left(A^{n}\right) \otimes \mathrm{F}=D\left(B^{n}\right) \otimes \mathrm{F}$ into a $B$-free resolution, using the auxiliary maps described in the next theorem:

THEOREM 7.1. Let $A$ be a ring, and let $M$ be an $A$-module which is annihilated by elements $x_{1}, \ldots, x_{n} \in A$. Suppose the ideal $\left(x_{1}, \ldots, x_{n}\right)$ contains a nonzero divisor. If $\mathbf{F}$ is a free resolution of $M$, then there are endomorphisms $s_{\alpha}$ of degree $2|\alpha|-1$ of $F$ as a graded module for each multi-index $\alpha$ satisfying

(i) $s_{0}$ is the differential of $\mathbf{F}$.

(ii) If $\alpha$ is the multi-index $\langle 0, \ldots, 0,1,0, \ldots, 0\rangle$ ( 1 in the jth place) then the map $s_{0} s_{\alpha}+s_{\alpha} s_{0}$ is multiplication by $x_{j}$.

(iii) If $\gamma$ is a multi-index with $|\gamma|>1$, then $\Sigma_{\alpha+\beta=\gamma} s_{\alpha} s_{\beta}=0$.

RemarKs. (1) If $F$ is finite, only finitely many $s_{\alpha}$ are nonzero.

(2) If $x_{1}, \ldots, x_{n}$ is a regular sequence on $A$, and if $\mathbf{K}$ is the Koszul complex resolving $\left(x_{1}, \ldots, x_{n}\right)$, considered as a differential graded algebra, then it seems reasonable to hope that the minimal free resolution $\mathbf{F}$ can be given the structure of a differential graded K-module. (For example, if $M$ were cyclic, and $\mathbf{F}$ had the structure of a graded commutative associative differential algebra conjectured in [B-E 1], this would follow. In any case, there are resolutions of any module $M$ which are K-modules [G2].) In plain terms, this means that for each $j=1, \ldots, n$, multiplication by $x_{j}$ on $\mathbf{F}$ should be homotopic to 0 by a map $s_{j}$ satisfying $s_{j}^{2}=0$, $s_{i} s_{j}=-s_{j} s_{i}$. If such maps exist for $\mathbf{F}$, then for each multi-index $\alpha$ we could set

$$
s_{\alpha}= \begin{cases}0 & \text { if } \alpha=0 \\ s_{j} & \text { if } \alpha=\langle 0, \ldots, 1, \ldots, 0\rangle \text { (with } 1 \text { in the } j \text { th place), } \\ 0 & \text { otherwise }\end{cases}
$$

and this choice would satisfy Theorem 7.1. Thus the theorem may be regarded as giving an "approximate K-module structure" on $\mathbf{F}$. See note at end of paper.

Using Theorem 6.1 , we can construct $A /\left(x_{1}, \ldots, x_{n}\right)$-free resolutions from $A$-free resolutions, if $x_{1}, \ldots, x_{n}$ is an $A$-sequence, as follows:

TheOREM 7.2. Let $A$ be $a$ ring, and let $x_{1}, \ldots, x_{n}$ be an $A$-sequence. Set $B=A /\left(x_{1}, \ldots, x_{n}\right)$, and let $\mathbf{F}$ be an $A$-free resolution of $a B$-module $M$. Let $\left\{s_{\alpha}\right\}$ be a family of endomorphisms of $\mathbf{F}$ as a graded module satisfying the conditions of Theorem 7.1. Finally, let $t_{1}, \ldots, t_{n}$ be variables of degree -2 , and set $\mathbf{D}=D\left(B^{n}\right)=$ $\operatorname{Hom}_{\text {graded } B \text {-modules }}\left(B\left[t_{1}, \ldots, t_{n}\right], B\right)$, with the natural structure of $a B\left[t_{1}, \ldots, t_{n}\right]-$ module. The graded $B$-module $\mathrm{D} \otimes \mathrm{F}$, equipped with the differential $\partial=\Sigma_{\alpha} t^{\alpha} \otimes s_{\alpha}$ is $a B$-free resolution of $M$. Moreover, $t_{j}\left(A,\left\{x_{j}\right\}, \mathbf{D} \otimes \mathbf{F}\right)$ may be chosen to be $t_{j} \otimes 1$.

REMARKs. (1) Write $\tau_{1}, \ldots, \tau_{n}$ for the dual basis to $t_{1}, \ldots, t_{n}$, so that $\tau^{(\alpha)}=$ $\tau_{1}^{\left(\alpha_{1}\right)} \cdots \tau_{n}^{\left(\alpha_{n}\right)}$ is a dual basis of $\mathbf{D}$ to the base of monomials. As in $\S 2$, we see that $t^{\alpha}\left(\tau^{(\beta)}\right)=0$ for all $\alpha$ with $|\alpha|>|\beta|$. Thus $\partial_{D \otimes F}$ is well defined even though the sum involved is formally infinite. 
(2) The construction of $\mathbf{D} \otimes \mathbf{F}$ generalizes the construction given in $\S 5$. If $\mathbf{F}$ has length 1 , that is

$$
\mathbf{F}: 0 \rightarrow G \underset{s_{0}}{\rightarrow} F
$$

then $s_{0}=\varphi$, and the only nontrivial $s_{\alpha}$ is $s_{1}: \dot{F} \rightarrow G$, which satisfies $s_{1} s_{0}=x$. Since $x$ is a nonzero divisor, this implies $s_{0} s_{1}=x$, so $\left(s_{0}, s_{1}\right)$ is a matrix factorization of $x$, and with the notation of $\S 5, \mathrm{D} \otimes \mathrm{F}=\mathrm{F}\left(s_{0}, s_{1}\right)$.

Proof of Theorem 7.1. Beginning with the definition $s_{0}=\partial$, we will construct the $s_{\alpha}$ by induction on $|\alpha|$.

Condition (ii) is the assertion that $s_{\alpha}$, for $\alpha=\langle 0, \ldots, 0,1, \ldots, 0\rangle$ (with 1 in the $j$ th place), is a homotopy for multiplication by $x_{j}$. Since $x_{j} M=0$, multiplication by $x_{j}$ is indeed homotopic to 0 on $\mathbf{F}$, so the existence of $s_{\alpha}$ for $|\alpha|=1$ satisfying condition (ii) is assured.

Now suppose $s_{\alpha}$ have been constructed for $\alpha$ with $|\alpha|<\left|\gamma_{0}\right|$, for some $\gamma_{0}$. Set

$$
\varepsilon=-\sum_{\substack{\alpha+\beta=\gamma_{0} \\|\alpha|<\gamma_{0} \\|\beta|<\gamma_{0}}} s_{\alpha} s_{\beta},
$$

we seek a map $s_{\gamma_{0}}$ with $s_{\gamma_{0}} s_{0}+s_{0} s_{\gamma_{0}}=\varepsilon$. Since $s_{0}^{2}=0$, any map of the form $s s_{0}+s_{0} s$ must commute with $s_{0}$. A straightforward but tedious computation, using (i), (ii) and (iii) with $|\gamma|<\left|\gamma_{0}\right|$, shows that, indeed, $\varepsilon s_{0}=s_{0} \varepsilon$. The next lemma thus finishes the proof.

LemMA 7.3. Let $\mathbf{F}$ be a free resolution of an $A$-module $M$, and suppose that the annihilator of $M$ contains a nonzero divisor. Let $\varepsilon: \mathbf{F} \rightarrow \mathbf{F}$ be an endomorphism of degree $k>0$ of $\mathbf{F}$ as a graded module, and let $s_{0}$ be the differential of $\mathbf{F}$. Then there exists an endomorphism $s$ of $\mathrm{F}$ as a graded module such that $s_{0} s+s s_{0}=\varepsilon$ if and only if $s_{0} \varepsilon=\varepsilon s_{0}$.

Proof of Lemma 7.3. The necessity of the condition is obvious; we prove the sufficiency. Since $\varepsilon: \mathbf{F} \rightarrow \mathbf{F}$ commutes with $s_{0}$, it is an endomorphism of $\mathbf{F}$ as a complex of degree $k>0$. But since $M$ is annihilated by a nonzero divisor, the induced map

$$
M=\operatorname{Coker}\left(F_{1} \rightarrow F_{0}\right) \rightarrow \operatorname{Coker}\left(F_{k+1} \rightarrow F_{k}\right) \subset F_{k-1}
$$

must be 0 . Thus $\varepsilon$ is homotopic to 0 ; that is, there exists a map such that $\varepsilon=s_{0} s+s s_{0}$.

Before launching into the proof of Theorem 7.2, we examine, under the hypotheses of Theorem 7.2, the homology of the complex $B \otimes \mathbf{F}$. If $\alpha=$ $\langle 0, \ldots, 0,1,0, \ldots, 0\rangle$ ( $i$ in the $j$ th place), we will write $s_{j}$ in place of $s_{\alpha}$ for the map making multiplication by $x_{j}$ homotopic to 0 . We continue to write $s_{0}$ for the differential of $\mathbf{F}$. Since $s_{j} \partial+\partial s_{j}=x_{j}$, the map $1 \otimes s_{j}: B \otimes \mathbf{F} \rightarrow B \otimes \mathbf{F}$ is an endomorphism of $B \otimes \mathrm{F}$ as a complex, and thus acts on $H_{*}(B \otimes \mathrm{F})$. 
LEMma 7.4. With hypotheses as in Theorem 7.2, the homology of $B \otimes \mathrm{F}$ is given by

$$
H_{m}(B \otimes F) \simeq \bigwedge^{m} B^{n} \otimes M .
$$

Furthermore, there is a basis $u_{1}, \ldots, u_{n}$ of $B^{n}$ such that, writing

$$
\sigma_{j}: \wedge B^{n} \rightarrow \wedge B^{n}
$$

for the map $\sigma_{j}: b \mapsto u_{j} \wedge b$, the map

$$
\left(\bigwedge^{m} B^{n}\right) \otimes M=H_{m}(B \otimes \mathrm{F}) \rightarrow H_{m+1}(B \otimes \mathrm{F})=\left(\stackrel{m+1}{\left.\bigwedge^{n} B^{n}\right) \otimes M}\right.
$$

induced by $1 \otimes s_{j}$ is $\sigma_{j} \otimes 1$.

Proof. Let

$$
\mathbf{K}: \mathbf{K}\left(x_{1}, \ldots, x_{n}\right): \ldots \stackrel{\kappa}{\rightarrow} \stackrel{2}{\wedge} A^{n} \stackrel{\kappa}{\rightarrow} A^{n} \stackrel{\kappa}{\rightarrow} A
$$

be the Koszul complex which resolves $B$ as an $A$-module, and let $\tilde{u}_{1}, \ldots, \tilde{u}_{n}$ be a basis for $A^{n}$ such that $\kappa\left(\tilde{u}_{j}\right)=x_{j}$. It is easy to check that the map

$$
\tilde{\sigma}_{j}: \wedge A^{n} \rightarrow \wedge A^{n}
$$

given by $\tilde{\sigma}_{j}(a)=\tilde{u}_{j} \wedge a$ satisfies $\kappa \tilde{\sigma}_{j}+\tilde{\sigma}_{j} \kappa=x_{j}$. Let $\pi_{1}$ and $\pi_{2}$ be the natural projections of complexes

$$
B \otimes \mathbf{F} \stackrel{\pi_{1}}{\leftarrow} \mathbf{K} \otimes \mathbf{F} \stackrel{\pi_{2}}{\rightarrow} \mathbf{K} \otimes M .
$$

Each of $\pi_{1}$ and $\pi_{2}$ induces an isomorphism on homology and factors through $B \otimes \mathbf{K} \otimes \mathbf{F}$. Moreover, the maps

$$
\delta_{j}=\tilde{\sigma}_{j} \otimes 1+1 \otimes s_{j}: \mathbf{K} \otimes \mathbf{F} \rightarrow \mathbf{K} \otimes \mathbf{F}
$$

satisfy $\left(\kappa \otimes s_{0}\right) \delta_{j}+\delta_{j}\left(\kappa \otimes s_{0}\right)=x_{j}$ for each $j$, and make the diagram of complexes

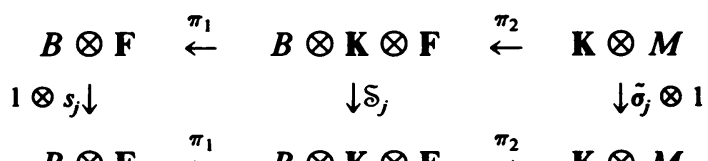

commute. Consequently, $1 \otimes s_{j}$ induces the same map as $\tilde{\sigma}_{j} \otimes 1$ on

$$
H_{*}(B \otimes \mathrm{F}) \cong \operatorname{Tor}_{*}^{A}(B, M) \simeq H_{*}(\mathrm{~K} \otimes M) .
$$

Now $H_{*}(K \otimes M)=\left(\bigwedge A^{n}\right) \otimes M=\bigwedge B^{n} \otimes M$, since $\kappa \otimes 1_{M}$ is 0 , so the map induced by $1 \otimes s_{j}$ on homology is $\sigma_{j} \otimes 1$.

Proof of Theorem 7.2. Let $D\left(A^{n}\right)=\operatorname{Hom}\left(A\left[t_{1}, \ldots, t_{n}\right], A\right)$, so that $\mathrm{D}=B \otimes$ $D\left(A^{n}\right)$, and define

$$
\tilde{\partial}_{\mathbf{D} \otimes \mathbf{F}}=\sum_{\alpha} t^{\alpha} \otimes s_{\alpha}: D\left(A^{n}\right) \otimes \mathbf{F} \rightarrow D\left(A^{n}\right) \otimes \mathbf{F},
$$

so that $\partial=B \otimes \tilde{\partial}$. We will continue to write $s_{0}$ for the differential of $F$, and $s_{j}$ for $s_{\alpha}$ when $\alpha=\langle 0, \ldots, 0,1,0, \ldots, 0\rangle$ (with a 1 in the $j$ th place). 
It follows easily from the formulas of Theorem 7.1 that

$$
\tilde{\partial}^{2}=\sum_{j=1}^{n} t_{j} \otimes x_{j}=\sum x_{j}\left(t_{j} \otimes 1\right),
$$

where we have written $x_{j}$ for the map "multiplication by $x_{j}$ ". Thus $\partial^{2}=B \otimes \tilde{\partial}^{2}=$ 0 , so $\mathrm{D} \otimes \mathrm{F}$ is a complex, and $t_{j}\left(A,\left\{x_{j}\right\}, \mathrm{D} \otimes \mathrm{F}\right)=B \otimes\left(t_{j} \otimes 1\right)=t_{j} \otimes 1$, as claimed.

It remains to prove that $D \otimes F$ is exact. We will do this by regarding $D \otimes F$ as a filtered complex, and considering the associated spectral sequence. (See, for example, [S].)

We filter $\mathbf{D} \otimes \mathbf{F}$ by degree in $\mathbf{D}=D\left(B^{n}\right)$, setting

$$
\mathscr{F}_{l}=\mathscr{F}_{l}(\mathbf{D} \otimes \mathbf{F})=\sum_{k<l} D_{k}\left(B^{n}\right) \otimes \mathbf{F}
$$

so that $0=\mathscr{F}_{0} \subset \mathscr{F}_{1} \subset \ldots \subset \mathbf{D} \otimes \mathbf{F}$. We also set

$$
\begin{aligned}
\operatorname{gr}_{l}(\mathbf{D} \otimes \mathbf{F}) & =\mathscr{F}_{l} / \mathscr{F}_{l-1}=D_{l}\left(D^{n}\right) \otimes \mathbf{F}, \\
\operatorname{gr}(\mathbf{D} \otimes \mathbf{F}) & =\sum \operatorname{gr}_{l}(\mathbf{D} \otimes \mathbf{F}) .
\end{aligned}
$$

Since each $t_{j}$ acts on $\mathbf{D}$ with degree $-2, \mathscr{F}_{l}$ is a subcomplex. Associated with this filtration of $\mathbf{D} \otimes \mathbf{F}$ is a spectral sequence

$$
E^{1}=H(\operatorname{gr}(\mathrm{D} \otimes \mathrm{F})) \Rightarrow H(\mathrm{D} \otimes \mathrm{F})
$$

We assert that the filtration is finite on each term of the complex $\mathbf{D} \otimes \mathbf{F}$, in fact

$$
\mathscr{F}_{i}\left[(\mathbf{D} \otimes \mathbf{F})_{m}\right]=\sum_{k<l ; 2 k+j=m} D_{k}\left(B^{n}\right) \otimes F_{j}=(\mathbf{D} \otimes \mathbf{F})_{m}
$$

if $l>m / 2$, since $F_{j}=0$ for $j<0$. Thus the above spectral sequence converges.

We next compute $E^{1}$ and its differential $d^{1}$. Since $D_{l}\left(B^{n}\right)$ is a free $B$-module, we have

$$
\begin{aligned}
H_{m}\left(\mathrm{gr}_{l} \mathrm{D} \otimes \mathrm{F}\right) & =H_{m}\left(D_{l}\left(B^{n}\right) \otimes \mathrm{F}\right)=D_{l}\left(B^{n}\right) \otimes H_{m}(B \otimes \mathrm{F}) \\
& =D_{l}\left(B^{n}\right) \otimes \bigwedge^{m} B^{n} \otimes M,
\end{aligned}
$$

by Lemma 7.4. The differential

$$
d^{1}: H_{m}\left(\mathrm{gr}_{l}(\mathrm{D} \otimes \mathrm{F})\right) \rightarrow H_{m+1}\left(\mathrm{gr}_{l-1}(\mathrm{D} \otimes \mathrm{F})\right)
$$

is induced by $\partial=\Sigma t^{\alpha} \otimes s_{\alpha}$, and is thus given by

$$
\sum_{j=1}^{n} t_{j} \otimes s_{j}: D_{l}\left(B^{n}\right) \otimes H_{m}(B \otimes \mathrm{F}) \rightarrow D_{l-1}\left(B^{n}\right) \otimes H_{m+1}(B \otimes \mathrm{F}) .
$$

By the second statement of Lemma 7.4, this is the same as

$$
\sum_{j=1}^{n} t_{j} \otimes \alpha_{1} \otimes 1: D_{l}\left(B^{n}\right) \otimes \bigwedge^{m} B^{n} \otimes M \rightarrow D_{l-1}\left(B^{n}\right) \otimes \bigwedge^{m+1} B^{n} \otimes M
$$

If we now define complexes

$$
\begin{gathered}
A_{r}\left(B^{n}\right): 0 \rightarrow D_{r}\left(B^{n}\right) \otimes \stackrel{0}{\wedge} B^{n} \stackrel{\varepsilon}{\rightarrow} D_{r-1}\left(B^{n}\right) \otimes \stackrel{1}{\wedge} B^{n} \rightarrow \\
\ldots \stackrel{\varepsilon}{\rightarrow} D_{\sigma-n}\left(B^{n}\right) \otimes \stackrel{n}{\wedge} B^{n} \rightarrow 0
\end{gathered}
$$


by $\varepsilon=\sum_{i=1}^{n} t_{i} \otimes \sigma_{i}$, we see that $E^{1}$, with its differential $d^{1}$, is the direct sum of the complexes $\mathbf{A}_{r}\left(B^{n}\right) \otimes M$.

The next lemma will show that the homology of all these complexes is 0 , except that $H\left(\mathrm{~A}_{0}\left(B^{n}\right) \otimes M\right) \cong M$. Thus the spectral sequence will degenerate at $E^{2}$, and we will have $H(\mathrm{D} \otimes \mathrm{F})=M$, so that $\mathrm{D} \otimes \mathrm{F}$ is a resolution of $M$. Thus Theorem 7.2 will be established by the following lemma:

LEMMA 7.5. With the above notation, the complex $\mathbf{A}_{r}\left(B^{n}\right)$ is split exact for all $r>0$. The homology of

$$
\mathrm{A}_{0}: 0 \rightarrow D_{0}\left(B^{n}\right) \otimes \stackrel{0}{\wedge} B^{n} \rightarrow 0
$$

is $B$.

Proof. The proof is given in full in [B-E 2]. The idea is that $\sum_{r=0}^{\infty} \mathbf{A}_{r}$ is the $B$-dual of the Koszul complex $\mathbf{K}^{B\left[t_{1}, \ldots, t_{n}\right]}$ which resolves

$$
B=B\left[t_{1}, \ldots, t_{n}\right] /\left(t_{1}, \ldots, t_{n}\right)
$$

over the polynomial ring $B\left[t_{1}, \ldots, t_{n}\right]$. Thus the homology of $\sum_{r=0}^{\infty} A_{r}$ is $B$ ("in degree $(0,0)$ "), and $A_{r}$ is exact for $r>0$. Split exactness follows from exactness, since $A_{r}$ is a $B$-free complex. The second statement of the lemma is obvious.

8. The inductive construction of minimal resolutions. In this section we will use Theorem 3.1, to give a sort of inductive construction of minimal resolutions over a complete intersection; we show that such a resolution has a truncation which is derived by the process of $\$ 7$ from a resolution over a complete intersection of smaller codimension.

Consider the setting of Theorem 3.1 where we have a local ring $A$, an $A$-sequence $x_{1}, \ldots, x_{n}$, and the factor ring $B=A /\left(x_{1}, \ldots, x_{n}\right)$. Let $B_{1}=A /\left(x_{2}, \ldots, x_{n}\right)$. If F is a $B$-free complex, then $t_{1}\left(A,\left\{x_{j}\right\}, F\right)=t_{1}\left(B_{1}, x_{1}, F\right)$. Our basic result is the following:

TheOREM 8.1. Let $B_{1}$ be a local ring, and let $x \in B_{1}$ be a nonzero divisor. Set $B=B_{1} /(x)$, and let $\mathrm{F}: \ldots \stackrel{\partial}{\rightarrow} F_{1} \stackrel{\partial}{\rightarrow} F_{0}$ be a $B$-free resolution of a $B$-module $M$ such that $t=t\left(B_{1}, x, F\right): F \rightarrow F$ is an epimorphism. Let

$$
\mathbf{F}^{\sharp}: \ldots \stackrel{\partial^{\sharp}}{\rightarrow} F_{1}^{\sharp} \stackrel{\partial^{\sharp}}{\rightarrow} F_{0}^{\#}
$$

be a sequence of maps of free $B_{1}$-modules such that $B \otimes \mathrm{F}^{\#}=\mathrm{F}$. If $\overline{\partial^{\sharp 2}}=x t^{\#}$, then

$$
\mathbf{K}^{\sharp}=\operatorname{Ker} t^{\#}: \ldots \stackrel{\partial^{\sharp} \mid K_{3}^{\sharp}}{\rightarrow} K_{2}^{\#} \stackrel{\partial^{\sharp} \mid K_{2}^{\sharp}}{\rightarrow} F_{1}^{\#} \stackrel{\partial^{*}}{\rightarrow} F_{0}^{\#}
$$

is a $B_{1}$-free resolution of $M$.

Moreover setting $\mathbf{D}=D\left(B^{1}\right)$ then $\mathbf{F} \cong \mathbf{D} \otimes \mathbf{K}^{\#}$ as complexes, with the differential on $\mathbf{D} \otimes \mathbf{K}^{\sharp}$ being that defined in Theorem 7.2.

REMARK. If $\mathbf{F}$ is minimal, then $\mathbf{K}^{\#}$ is too. 
COROllary 8.2. Let $B$ be a local complete intersection of codimension $n$, and let $\mathbf{F}$ be the minimal $B$-free resolution of a $B$-module $M$. There exist a local complete intersection $B_{1}$ of codimension $n-1$ and an element $x \in B_{1}$ such that $B=B_{1} /(x)$, and for all large $N$ the truncation $\mathbf{F}^{(N)}$ of $\mathbf{F}$ has the form $\mathbf{F}^{(N)} \simeq \mathbf{D} \otimes \mathbf{G}$, where $\mathbf{D}=D\left(B^{1}\right)$ is the divided power algebra, $\mathbf{G}$ is the minimal $B_{1}$-free resolution of the $N$ th $B$-syzygy of $M$, and $\mathbf{D} \otimes \mathbf{G}$ has the differential defined in Theorem 7.2.

Proof of Corollary 8.2. Apply Theorems 3.1 and 8.1.

Part of Theorem 8.1 actually holds more generally:

LEMmA 8.3. Let $B_{1}$ be a local ring, and let $x \in B_{1}$ be a nonzero divisor. Set $B=B_{1} /(x)$ and let $(\mathbf{F}, \partial)$ be a complex of free $B$-modules. Let $\left(F^{\#}, \partial^{\#}\right)$ be a lifting of (F, d) to a sequence of maps of free $B$-modules, as in Theorem 8.1. If $t^{\#}: F^{\#} \rightarrow F^{\#}$ satisfies $x t^{\#}=\partial^{\# 2}$, then $t^{\#}$ commutes with $\partial^{\#}$ and $\operatorname{Ker} t^{\#}$ is a complex with differential $\partial \# \mid \operatorname{Ker} t^{\#}$.

Proof. $x t^{\#}=\partial^{\# 2}$ clearly commutes with $\partial^{\#}$; since $x$ is a nonzero divisor, $t^{\#}$ does too. This implies in particular that $\partial^{\sharp}\left(\operatorname{Ker} t^{\sharp}\right) \subset \operatorname{Ker} t^{\sharp}$. Also, $\partial^{\# 2}\left(\operatorname{Ker} t^{\sharp}\right)=$ $x t^{\#}\left(\operatorname{Ker} t^{\#}\right)=0$, so the second statement follows from the first.

Proof of Theorem 8.1. By Nakayama's Lemma, $t^{\#}: F^{\#} \rightarrow F^{\#}$ is an epimorphism. Thus for each $i$,

$$
K_{i}^{\#}=\operatorname{Ker}\left(t^{\#}: F_{i}^{\#} \rightarrow F_{i-2}^{\#}\right)
$$

is a free summand of $F_{i}^{\sharp}$. Note that for $i=0,1, K_{i}^{\#}=F_{i}^{\sharp}$. Thus by Lemma 8.3, $\mathbf{K}^{\sharp}$ is a free complex.

We must now show

(i) $\operatorname{Coker}\left(F_{1}^{\sharp} \stackrel{\partial}{\rightarrow} F_{0}^{\sharp}\right)=M$,

(ii) $H_{i}\left(\mathbf{K}^{\sharp}\right)=0$ for $i>0$.

For (i), note that since $t_{1}^{\#}: F_{2}^{\#} \rightarrow F_{0}^{\#}$ is onto, we have

$$
x F_{0}^{\#}=\text { image } x t_{1}^{\sharp}=\text { image } \partial^{\sharp 2} \subset \operatorname{image}\left(F_{1}^{\sharp} \stackrel{\partial^{\sharp}}{\rightarrow} F_{0}^{\sharp}\right) \text {. }
$$

Thus $\operatorname{Coker}\left(F_{1}^{\sharp} \rightarrow F_{0}^{\sharp}\right)$ is annihilated by $x$. Thus

$$
\operatorname{Coker}\left(F_{1}^{\sharp} \rightarrow F_{0}^{\sharp}\right)=B \otimes \operatorname{Coker}\left(F_{1}^{\sharp} \rightarrow F_{0}^{\sharp}\right)=\operatorname{Coker}\left(F_{1} \rightarrow F_{0}\right)=M \text {. }
$$

For (ii), let $K=B \otimes K^{\#}$. Since $K^{\sharp}$ is a free direct summand of $F^{\#}$, we will have $\mathbf{K}=\operatorname{Ker} t$. Thus there is an exact sequence of complexes $0 \rightarrow \mathbf{K} \rightarrow \mathbf{F} \stackrel{t}{\rightarrow} \mathbf{F} \rightarrow 0$ (where degree $t=-2$ as usual). From the ensuing long exact sequence in homology, we easily deduce

$$
\begin{aligned}
& H_{i}(\mathbf{K})=0, \quad i \geqslant 2, \\
& H_{1}(\mathbf{K}) \cong H_{0}(\mathrm{~K}) \cong H_{0}(\mathrm{~F})=M .
\end{aligned}
$$

Next consider the exact sequence of complexes $0 \rightarrow K^{\sharp} \stackrel{x}{\rightarrow} K^{\sharp} \rightarrow K \rightarrow 0$. The long exact sequence in homology together with $(*)$ yields exact sequences

$$
0 \rightarrow H_{i}\left(\mathbf{K}^{\sharp}\right) \stackrel{x}{\rightarrow} H_{i}\left(\mathbf{K}^{\sharp}\right) \rightarrow 0, \quad i>2,
$$


and

$$
0 \rightarrow H_{1}\left(\mathbf{K}^{\sharp}\right) \stackrel{x}{\rightarrow} H_{1}\left(\mathbf{K}^{\sharp}\right) \rightarrow M \stackrel{\delta}{\rightarrow} H_{0}\left(\mathbf{K}^{\sharp}\right) \stackrel{x}{\rightarrow} H_{0}\left(\mathbf{K}^{\sharp}\right) \rightarrow M \rightarrow 0 .
$$

From (i) we get $H_{0}\left(\mathrm{~K}^{\sharp}\right) \cong M$ so (***) becomes

$$
0 \rightarrow H_{1}\left(\mathrm{~K}^{\sharp}\right) \stackrel{x}{\rightarrow} H_{1}\left(\mathrm{~K}^{\sharp}\right) \rightarrow \stackrel{\delta}{\rightarrow} M \rightarrow 0 .
$$

Since an endomorphism of a noetherian module which is an epimorphism must be an isomorphism, ker $\delta=0$ and we obtain $0 \rightarrow H_{1}\left(K^{\sharp}\right) \stackrel{x}{\rightarrow} H_{1}\left(K^{\sharp}\right) \rightarrow 0$. Since $x$ is in the maximal ideal of $B_{1}$, this last sequence and $(* *)$ show that $H_{i}\left(\mathbf{K}^{\sharp}\right)=0$ for all $i \geqslant 1$ by Nakayama's Lemma. This proves (ii).

It remains to show that $\mathbf{F} \cong \mathbf{D} \otimes \mathbf{K}^{\sharp}$. However, by Theorem 7.1, both are $B$-free resolutions of the same $B$-module $M$. Since $K_{i}^{\sharp}=\operatorname{Ker}\left(F_{i}^{\sharp} \stackrel{t}{\rightarrow} F_{i-2}^{\sharp}\right)$, we have

$$
\operatorname{rank} K_{i}^{\#}=\operatorname{rank} F_{i}-\operatorname{rank} F_{i-2}, \quad i>0 .
$$

An easy computation now shows that $\operatorname{rank} F_{i}=\operatorname{rank}\left(\mathbf{D} \otimes \mathbf{K}^{\sharp}\right)_{i}$. Since both $\mathbf{F}$ and $\mathbf{D} \otimes K^{\#}$ must be built out of the minimal $B$-free resolution of $M$ by the addition of various trivial summands, this implies $\mathbf{F} \cong \mathbf{D} \otimes \mathbf{K}^{\sharp}$.

9. Two results on modules over finite groups. Modular representations of finite groups behave in many ways like modules over complete intersections, probably because, in characteristic $p$, the subalgebra corresponding to a maximal abelian $p$ subgroup is a complete intersection (see the proof of Theorem 9.1 for details); however, the nonabelian $p$-part, and the whole $p^{\prime}$-part, make it difficult to apply our theory directly except in special cases.

The proof of Theorem 9.1, below, is such a case. On the other hand, our proof of Theorem 9.2 is really an application of the philosophy of this paper, rather than of the results we have developed. Both results are extensions of results of J. Alperin [A2]; in the case of Theorem 9.1 we get a better bound (as conjectured by Alperin), while in Theorem 9.2 we are able to drop Alperin's assumption that the ground field $k$ is algebraic over the prime field.

Theorem 9.1 has been proved, independently and by very different means, by Carlson [C]. The interested reader should consult his paper for a more precise analysis of the group-theoretic case.

THEOREM 9.1. Let $k$ be a field of characteristic $p \neq 0$, and let $G$ be a finite group. Suppose $G$ contains an abelian subgroup of order $p^{n}$ and exponent $p^{e}$. If $M$ is a $k G$-module that can be resolved by $k G$-projections of bounded rank, then $p^{n-e} \mid \operatorname{dim}_{k} M$.

Proof. Of course we may assume that $G$ is abelian of rank $p^{n}$ and exponent $p^{e}$; that is, in terms of generators and relations, $G=\left\langle g_{1}, \ldots, g_{s} ; g_{1}^{p^{e}}=g_{2}^{p^{n_{2}}}=\cdots=\right.$ $\left.g_{s}^{p^{n_{3}}}=1\right\rangle$, with $n_{i} \leqslant e$ and $e+\sum_{i=2}^{s} n_{i}=n$. Writing $x_{i}=g_{i}-1$, we see that $k G=k\left[\left|X_{1}, \ldots, X_{s}\right|\right] /\left(X_{1}^{p^{e}}, X_{2}^{p^{n_{2}}}, \ldots, X_{s}^{p^{n_{s}}}\right)$. Since $k G$ is thus a complete intersection, Theorem 4.1 shows that the resolution of $M$ becomes eventually periodic. Since $k G$ is self-injective, $M$ is a direct sum of a free module and a module whose 
resolution is periodic. Since free modules have rank $p^{n}$, we may assume that $M$ itself has a periodic resolution.

Extending $k$ if necessary to an algebraically closed field, and applying Theorem 5.2 (together with the explicit construction of the ring $B_{1}$ in the proof of that theorem) we see that there are elements $r_{2}, \ldots, r_{s} \in k$ such that $M$ has projective dimension 1 as a module over

$$
k\left[\left|X_{1}, \ldots, X_{n}\right|\right] /\left(X_{2}^{p^{n_{2}}}-r_{2} X_{1}^{p^{e}}, \ldots, X_{s}^{p^{n_{s}}}-r_{s} X_{1}^{p^{c}}\right) .
$$

Note that if $t_{i}=r^{1 / p^{n_{i}}}$ then

$$
X_{i}^{p^{n_{i}}}-r_{i} X_{1}^{p^{e}}=\left(X_{i}-t_{i} X_{1}^{p^{e-n_{i}}}\right)^{p^{n_{i}}},
$$

since $n_{i} \leqslant e$. This suggests the change of variable $y_{1}=X_{1}$,

$$
y_{i}=X_{i}-t_{i} X_{1}^{p^{e-n_{i}}}, \quad i=2, \ldots, s,
$$

and in this notation, $M$ is a module of projective dimension 1 over the ring $k\left[\left|y_{1}, \ldots, y_{s}\right|\right] /\left(y_{2}^{p_{2}}, \ldots, y_{s}^{p^{n_{s}}}\right)$. We now take any matrix presenting $M$, lift it to a matrix over $k\left[\left|y_{1}, \ldots, y_{s}\right|\right]$, and let $\tilde{M}$ be the cokernel of the lifted matrix. Since $\tilde{M}$ is complete and separated for the $\left(y_{2}, \ldots, y_{s}\right)$-adic topology, and

$$
\tilde{M} /\left(y_{2}, \ldots, y_{s}\right) \tilde{M}=M
$$

has finite length, we see that $\tilde{M}$ is finitely generated over the subring $k\left[\left|y_{2}, \ldots, y_{n}\right|\right]$. On the other hand, it is easy to show that $y_{2}, \ldots, y_{n}$ is an $\tilde{M}$-regular sequence (see for example [B-E 0]). By the Auslander-Buchsbaum-Serre Theorems, [Mat, p. 113] $\tilde{M}$ is then a free $k\left[\left[y_{2}, \ldots, y_{s}\right]\right]$-module, of rank $t$, say. But then

$$
M=\tilde{M} / y_{2}^{p^{n_{2}}}, \ldots, y_{s}^{p_{s}^{n_{s}}}
$$

has dimension $t p^{n_{2}} p^{n_{3}} \ldots p^{n_{s}}=t p^{n-e}$, as desired.

THEOREM 9.2. Let $k$ be a field, and let $G$ be a finite group. If $M$ is a finitely generated $k G$-module with no free summands, and if $M$ can be resolved by $k G$-projective modules of bounded rank, then $M$ has a periodic $k G$-resolution.

Proof. Let $J=\operatorname{rad} k G$. The ring $H^{*}(G, k)$ is noetherian by Evens Theorem [EV]. If $\mathbf{P} \rightarrow k$ is a projective resolution of $k$, then $\mathbf{P} \otimes M \rightarrow k \otimes M=M$ is a projective resolution of $M$, so $H^{*}(G, N)$ is a finitely generated graded $H^{*}(G, k)$ module.

If $H^{*}(G, N) \neq 0$ for infinitely many $i$, then, by standard theorems about primary decomposition, there will, for some large $n$, be an element $s \in H^{n}(G, k)$ such that for all large $i$, multiplication by $s$ induces a monomorphism $H^{i}(G, N) \rightarrow$ $H^{i+n}(G, N)$. Of course, $s$ is represented by a map of degree $-n$ from $\mathbf{P}$ to $\mathbf{P}$, and so the map $s$ induced on $H^{*}(G, N)$ may be represented as a map of degree $-n$ from $\mathbf{P} \otimes N$ to $\mathbf{P} \otimes N$. Of course, any other resolution of $N$ must also support a map of degree $-n$ inducing $s$.

Applying this to the module $M \otimes k G / J$, we see that there is an endomorphism of degree $-n$ of the minimal resolution $Q$ of $M$ which induces a monomorphism 
on the homology

$$
\operatorname{Ext}_{k G}^{i}(M, k G / J)=H^{i}\left(G, M \otimes_{k} k G / J\right),
$$

or, for large $i$, equivalently, induces epimorphisms $\operatorname{Tor}_{i+n}^{k G}(M, k G / J) \rightarrow$ $\operatorname{Tor}_{i}(M, k G / J)$ for all large $i$.

Since $Q$ is minimal, this implies that the maps

$$
s: Q_{n+i} \rightarrow Q_{i}
$$

are epimorphisms for all large $i$; since the ranks of the $Q_{i}$ are bounded, this implies that $s$ is actually an isomorphism, at least when restricted to a sufficiently high truncation of the complex $Q$, and thus the resolution of $M$ is eventually periodic, of period $n$. Since, however, $k G$ is self-injective, and since $M$ has by hypothesis no free summands, this shows that the resolution of $M$ is itself periodic of period $n$.

NOTE ADDED IN PROOF (March 1980). L. Avramov, in a recent preprint (to appear in Amer. J. Math.), has shown that there is a nonvanishing obstruction to the hope expressed in Remark 2 after Theorem 7.1, above. However, as he remarks, it is still possible to hope that that hope is fulfilled for some sequences $x_{1}, \ldots, x_{n}$.

\section{REFERENCES}

[A1] J. Alperin, Resolutions for finite groups, Internat. Sympos. on Theory of Finite Groups (Sapporo, Japan, 1974).

[A2] __ Periodicity in groups, Illinois J. Math. (to appear).

[B] H. Bass, On the ubiquity of Gorenstein rings, Math. Z. 82 (1963), 8-28.

[Bou] N. Bourbaki, Algèbre, Chapitres I-III, Hermann, Paris, 1970.

[B-E 0] D. A. Buchsbaum and D. Eisenbud, Lifting modules and a theorem on finite free resolutions, Ring Theory, Academic Press, New York, 1972.

[B-E 1] _ Algebra structures on resolutions, and some structure theorems for ideals of height 3, Amer. J. Math. (1977).

[B-E 2] _ Generic free resolutions and a family of generically perfect ideals, Advances in Math. (1976).

[B-E 3] __, What annihilates a module?, J. Algebra 47 (1977), 231-243.

[C] J. Carlson, The dimensions of periodic modules over modular group algebras, Illinois J. Math. 23 (1979), 295-306.

[EGA] A. Grothendieck, Eléments de géométrie algébrique, Chapitre IV, part 2, Inst. Hautes Études Sci. Publ. Math. 24 (1965).

[EV] L. Evens, The cohomology ring of a finite group. Trans. Amer. Math. Soc. 101 (1961), 224-239.

[G1] T. Gulliksen, $A$ proof of the existence of minimal $R$-algebra resolutions, Acta Math. 120 (1968).

[G2] _ A change of rings theorem, with application to Poincaré series and intersection multiplicity, Math. Scand. 34 (1974), 167-183.

[G-L] T. Gulliksen and G. Levin, Homology of local rings, Queen's Papers in Pure and Appl. Math., vol. 20, Queen's Univ., Kingston, Ontario, 1969.

[Kap] I. Kaplansky, Commutative rings, 2nd ed., University of Chicago Press, Chicago, Ill., 1974.

[L-V] G. Levin and W. Vasconcelos, Homological dimensions and Macaulay rings, Pacific J. Math. 25 (1968).

[Mat] H. Matsumura, Commutative algebra, Benjamin, New York, 1970.

[M] V. Mehta, Thesis, University of California, Berkeley, Calif., 1976.

[Nag] M. Nagata, Local rings, Interscience, New York, 1962.

[Nas] H.-J. Nastold, Zur Serreschen Multiplizitätstheorie in der arithmetischen Geometrie, Math. Ann. 143 (1961), 333-343.

[S] J. P. Serre, Algèbre local-multiplicités, 3rd ed., Lecture Notes in Math., vol. 11, Springer-Verlag, New York, 1975.

[Sh] J. Shamash, The Poincaré series of a local ring. I, J. Algebra 12 (1969), 453-470.

[Tate] J. Tate, Homology of Noetherian rings and local rings, Illinois J. Math. 1 (1957), 14-27.

Department of Mathematics, Brandeis University, Waltham, MassachusetTs 02254 